\title{
Significant prognostic values of nuclear genes encoding mitochondrial complex I subunits in tumor patients
}

\author{
L. D. $\mathrm{LI}^{1,2, \neq}$, H. F. SUN ${ }^{1,2, \neq}$, Y. BAI ${ }^{3, \ddagger}$, S. P. GAO ${ }^{1,2}$, H. L. JIANG ${ }^{1,2}$, W. JIN ${ }^{1,2, *}$ \\ ${ }^{1}$ Department of Breast Surgery, Key Laboratory of Breast Cancer in Shanghai, Collaborative Innovation Center of Cancer Medicine, Fudan Uni- \\ versity Shanghai Cancer Center, Shanghai, 200030, China; ${ }^{2}$ Department of Oncology, Shanghai Medical College, Fudan University, Shanghai, \\ 200030, China; ${ }^{3}$ Department of Obstetrics and Gynecology of Shanghai Medical College, Fudan University, Shanghai 200032
}

*Correspondence: weijin7207@126.com

${ }^{*}$ Contributed equally to this work.

Received December 15, 2015 / Accepted February 5, 2016

\begin{abstract}
In cancer biology, it remains still open question concerning the oncogenic versus oncosuppressor behavior of metabolic genes, which includes those encoding mitochondrial complex I(CI) subunits. The prognostic value of nuclear genome mRNAs expression of CI subunits is to be evaluated in the tumor patients. We used the Kaplan Meier plotter database, the cBio Cancer Genomics Portal, and the Oncomine in which gene expression data and survival information were from thousands of tumor patients to assess the relevance of nuclear genome mRNAs level of CI subunits to patients' survival, as well as their alterations in gene and expression level in tumors. We presented that the relative expression level of overwhelming majority of the nuclear genes of CI subunits with survival significance (overall survival, relapse free survival, progression free survival, distant metastasis free survival, post progression survival, and first progression), had consistent effects for patients in each type of four tumors separately, including breast cancer, ovarian cancer, lung cancer, and gastric cancer. However, in gene level, frequent cumulative or individual alteration of these genes could not significantly affect patients' survival and the overexpression of the individual gene was not ubiquitous in tumors versus normal tissues. Given that reprogrammed energy metabolism was viewed as an emerging hallmark of tumor, thus tumor patients' survival might potentially to be evaluated by certain threshold for overall expression of CI subunits. Comprehensive understanding of the nuclear genome encoded CI subunits may have guiding significance for the diagnosis and prognosis in tumor patients.
\end{abstract}

Key words: mitochondrial complex I, genes, tumor, prognosis, KM plotter

According to the United States Cancer Statistics, as the second leading cause of death in the United States, cancer is expected to be the leading cause of death in the next few years [1].

With high heterogeneity and complexity, despite advances in basic and clinical research, prognosis of malignant tumors remains discouraging due to the high postoperative recurrence rate and metastasis [2]. Therefore, the mechanism investigation for tumor incidence and progression, as well as tumor biomarkers identification are imperative and will help

Abbreviations: NADH - Nicotinamide Adenine Dinucleotide Hydrogen; OXPHOS - Oxidative Phosphorylation; OS - Overall Survival; ROS - Reactive Oxygen Species; CI - Mitochondrial Complex I; mtDNA: mitochondrial DNA; nDNA - nuclear DNA to provide better prognostic prediction and individualized treatments for patients.

As was indicated in an analysis of mitochondrial DNA (mtDNA) mutations in the literatures scanned in Medline from 1998 to 2011, in 921 tumor cases where the entire mitochondrial genome had been sequenced, about $56 \%$ of all tumors contained at least one mutation, with $28 \%$ being in mitochondrial complex I (CI) and 35\% in the Dloop [3]. Lu and colleagues reported similar results of mutations in mtDNA encoding CI subunits in a wide range of cancers [4].

Albeit frequent mtDNA mutations being documented in cancer, [5] it is still elusive as to what a role they play in tumorigenesis, and how mitochondrial function can be affected by a specific mtDNA mutation type. Notably, none of the known pathogenic point mutations causing 
primary mitochondrial disease is demonstrably associated with tumor [6]. Only recently the concept of heteroplasmy and threshold effect has been introduced to investigate the mtDNA mutations' functional consequences during cancer progression $[7,8]$.

Pelicano et al hold the view that mtDNA mutations result in downregulation of oxidative phosphorylation (OXPHOS) function, and thus promote tumor survival [9]. As the first and crucial component of mitochondrial respiratory chain, human CI comprises forty nine subunits. Forty two of them are encoded by the nuclear genome and assembled within both the hydrophilic and the hydrophobic arms [10]. While the remaining seven are encoded by mtDNA and constitute the hydrophobic arm, and together with the other seven nuclear gene-encoded ones, they constitute the evolutionarily conserved "core subunits" of CI, which are involved in the electron transfer and proton pumping. Whereas the rest "accessory" subunits may participate in stabilizing CI, regulating the enzymatic activity, preventing the generation of reactive oxygen species (ROS) or protecting the CI from oxidative damage $[11,12]$.

Unlike the mtDNA, being affected by their distinct structure, replication and hereditary mode, as well as the hazardous environment, nuclear DNA (nDNA) encoding the mitochondria components are more stable. And far few studies had specially focused on mitochondria nDNA in tumor prognosis.

In the current study, for the first time we investigated the prognostic value of individual nuclear genes encoding CI subunits ('all genes' for short where appears following the passage), especially focusing on the seven core subunits genes ('core genes' for short where appears following the passage) as the representative, in different four types of tumor patients, using Kaplan Meier plotter (KM plotter) database. Then the gene-level and expression-level alterations of the 'all genes' and 'core genes' were explored through the cBio Cancer Genomics Portal and Oncomine. Furthermore, the relationship among their survival significances, alterations in gene and expression levels and the tumor reprogrammed energy metabolism was discussed.

\section{Materials and methods}

The Kaplan Meier plotter. The Kaplan Meier plotter (http:// kmplot.com/analysis/) was capable to assess the effect of 54675/22277 genes on survival using 10188 cancer samples, which included 4142 breast, 1648 ovarian, 2437 lung and 1065 gastric cancer patients with a mean follow-up of 69/40/49/33 months. The background database was established using gene expression data and patients survival information from the Cancer Biomedical Informatics Grid (caBIG, http://cabig. cancer.gov/), Gene Expression Omnibus (GEO, http://www. ncbi.nlm.nih.gov/geo/), Cancer Genome Atlas (TCGA, http:// cancergenome.nih.gov), and the European genome-phenome archive (EGA, https://www.ebi.ac.uk/ega/), which was handled by a MySQL server and integrates gene expression and clinical data simultaneously [13-16].

In order to analyze the prognostic value of a particular gene, the cohorts were divided into two groups according to the median (or upper/lower quartile) expression of the gene. The two groups could be compared in terms of overall survival (OS), and relapse free survival (RFS), and so on [14].

Briefly, the forty-two 'all genes' (NDUFA1, NDUFA10, NDUFA11, NDUFA12, NDUFA13, NDUFA2, NDUFA3, NDUFA4, NDUFA5, NDUFA6, NDUFA7, NDUFA8, NDUFA9, NDUFAB1, NDUFAF1, NDUFAF2, NDUFAF3, NDUFAF4, NDUFB1, NDUFB10, NDUFB11, NDUFB2, NDUFB3, NDUFB4, NDUFB5, NDUFB6, NDUFB7, NDUFB8, NDUFB9, NDUFC1, NDUFC2, NDUFS1, NDUFS2, NDUFS3, NDUFS4, NDUFS5, NDUFS6, NDUFS7, NDUFS8, NDUFV1, NDUFV2, and NDUFV3) were individually entered into the database (http://kmplot.com/analysis/) respectively to obtain KaplanMeier survival plots in which the number-at-risk was indicated below the main plot. Hazard ratio (and $95 \%$ confidence intervals) and $\log$ rank $P$ value were calculated and displayed on the webpage.

The cBio Cancer Genomics Portal. The cBio Cancer Genomics Portal (http://cbioportal.org), developed at Memorial Sloan-Kettering Cancer Center (MSKCC), was specifically designed to address the unique data integration issues posed by large-scale cancer genomics projects, including the Cancer Genome Atlas (TCGA) and the International Cancer Genome Consortium (ICGC) [17]. It provided a web resource for exploring, visualizing, and analyzing multidimensional cancer genomics data, and presents us with graphical summaries of gene-level data from multiple platforms, network visualization and analysis, survival analysis, patient-centric queries, and software programmatic access.

We queried cross-cancer alteration summary for multigene cumulative alteration as well as individual gene changes in 105 cancer studies through the cBio Cancer Genomics Portal (www.cbioportal.org) to investigate the gene-level alterations of CI subunits in cancers.

Oncomine analysis. Oncomine, a cancer microarray database and web-based data-mining platform aimed at facilitating discovery from genome-wide expression analyses, in which differential expression analyses comparing most major types of cancer with respective normal tissues as well as a variety of cancer subtypes and clinical-based and pathology-based analyses were available for exploration [18].

The individual gene of 'all genes' expression level was analyzed using Oncomine. We compared mRNA levels of cancer vs. normal patient datasets. We selected 1.5 fold change, $P$ value $=0.05$, and top $10 \%$ gene rank as thresholds .

\section{Results}

Significant and consistent prognostic value of individual nuclear genes encoding CI core subunits in different cancer patients' survival. The $\mathrm{CI}$ was divided into six sub-complexes, 
and their 49 subunits were grouped to comprise their corresponding parts. The characteristics of the seven core subunits encoded by nDNA were shown in (S-Table1 and S-Table2) [9].

Among all the 49 Mitochondrial CI subunits' genes, only the 7 mitochondrial genes were not found in the Kaplan Meier plotter (http://kmplot.com/), probably due to the lack of mtDNA data compared to the nDNA.

We first examined the OS of the seven 'core genes' mRNA expression in www.kmplot.com, for breast cancer, ovarian cancer, lung cancer and gastric cancer respectively. Survival curves were plotted for all patients (Figures 1-3). The desired Affymetrix ID, Cases Number, Hazard Ratio (HR; and 95\% confidence intervals) and Log Rank $P$ were summarized in Table1.

For breast cancer, high expression of the 'core genes' mRNA except for NDUFS3 and NDUFV2 was found to be correlated to worse $\mathrm{OS}$ in all breast cancer patients followed for 25 years (Figure 1A, 1B, Figure 2A, 2B, 2C). While high level of NDUFV2 mRNA was found to be in relation with better OS, and NDUFS3 expression level had no significant OS for all breast cancer patients (Figure 1C; 3A). Integrated with the other 'all genes' in S-Table4, it could be indicated that 24 out of the 42 'all genes' had worse, and 2 better OS for all breast cancer patients (Figure 3E). Moreover, most of the "core genes" had worse OS in luminal B breast cancer patients (S-Table3.1). And, for pathology grade I and/or II patients, majority of the "core genes" predicted worse OS (S-Table3.2).

For ovarian cancer, high expression of NDUFS3, NDUFS7, NDUFS8 and NDUFV1 mRNA were found to be correlated to better OS in all ovarian cancer patients (Figure 1C; Figure 2A-C) However, NDUFS1 and NDUFS2 had the opposite effect. The Figure 3B showed that NDUFV2 expression had no significant OS for all ovarian cancer patients. Together with the other 'all genes' in S-Table4, it could be found that 15 out of the 42 'all genes' had worse, and 6 better OS for ovarian cancer patients (Figure 3E). Additionally, for the pathology grade I and/or II ovarian cancer patients, most of the "core genes" had significant prognostic values, better or worse (S-Table3.3). For serous ovarian cancer patients, almost all the "core genes" expression level could predicted better OS (S-Table3.4).

Table 1. The summary of desired Affymetrix ID, Cases Number, Hazard Ratio (HR; and 95\% confidence intervals) and Log Rank P of the individual core gene's OS curves for the four different tumors.

\begin{tabular}{|c|c|c|c|c|c|c|c|c|}
\hline Cancer & Subunits & Affymetrix ID & Survival & Cases-low & Cases-high & HR & $95 \% \mathrm{CI}$ & P-value \\
\hline \multirow[t]{7}{*}{ Breast cancer } & NDUFS1 & 203039_s_at & OS & 306 & 811 & 1.44 & $1.09-2.01$ & 0.011 \\
\hline & NDUFS2 & 208969_at & OS & 547 & 570 & 1.3 & $1.03-1.65$ & 0.029 \\
\hline & NDUFS3 & 201740_at & OS & 724 & 393 & 1.2 & $0.94-1.52$ & 0.15 \\
\hline & NDUFS7 & 211752_s_at & OS & 519 & 598 & 1.35 & $1.06-1.72$ & 0.014 \\
\hline & NDUFS8 & 203190_at & OS & 408 & 709 & 1.31 & $1.01-1.68$ & 0.039 \\
\hline & NDUFV1 & 208714_at & OS & 527 & 590 & 1.38 & $1.08-1.76$ & 0.0096 \\
\hline & NDUFV2 & 202941_at & OS & 417 & 700 & 0.73 & $0.57-0.93$ & 0.0097 \\
\hline \multirow[t]{7}{*}{ Ovarian cancer } & NDUFS1 & 203039_s_at & OS & 503 & 1079 & 1.24 & $1.07-1.43$ & 0.004 \\
\hline & NDUFS2 & 201966_at & OS & 1182 & 400 & 1.26 & $1.09-1.46$ & 0.0023 \\
\hline & NDUFS3 & 201740_at & OS & 934 & 648 & 0.84 & $0.73-0.97$ & 0.014 \\
\hline & NDUFS7 & 211752_s_at & OS & 459 & 1123 & 0.85 & $0.73-0.98$ & 0.025 \\
\hline & NDUFS8 & 203189_s_at & OS & 609 & 973 & 0.85 & $0.75-0.98$ & 0.021 \\
\hline & NDUFV1 & 208714_at & OS & 491 & 1091 & 0.84 & $0.73-0.97$ & 0.017 \\
\hline & NDUFV2 & 202941_at & OS & 428 & 1154 & 0.88 & $0.77-1.02$ & 0.098 \\
\hline \multirow[t]{7}{*}{ Lung cancer } & NDUFS1 & 203039_s_at & OS & 1339 & 587 & 0.88 & $0.77-1.01$ & 0.069 \\
\hline & NDUFS2 & 201966_at & OS & 1304 & 622 & 1.21 & $1.07-1.38$ & 0.036 \\
\hline & NDUFS3 & 201740_at & OS & 524 & 1402 & 1.52 & $1.3-1.77$ & $1.10 \mathrm{E}-07$ \\
\hline & NDUFS7 & 211752_s_at & Os & 624 & 1302 & 1.27 & $1.1-1.47$ & 0.00093 \\
\hline & NDUFS8 & 203189_s_at & OS & 482 & 1444 & 1.76 & $1.48-2.09$ & $6.90 \mathrm{E}-11$ \\
\hline & NDUFV1 & 208714_at & OS & 1441 & 485 & 1.28 & $1.12-1.48$ & 0.0047 \\
\hline & NDUFV2 & 202941_at & OS & 623 & 1303 & 1.64 & $1.41-1.9$ & $6.90 \mathrm{E}-11$ \\
\hline \multirow[t]{7}{*}{ Stomach cancer } & NDUFS1 & 203039_s_at & OS & 623 & 253 & 0.5 & $0.4-0.61$ & $4.00 \mathrm{E}-11$ \\
\hline & NDUFS2 & 201966_at & Os & 325 & 551 & 0.69 & $0.58-0.82$ & $1.70 \mathrm{E}-05$ \\
\hline & NDUFS3 & 201740_at & OS & 538 & 348 & 0.66 & $0.55-0.78$ & $3.40 \mathrm{E}-06$ \\
\hline & NDUFS7 & 211752_s_at & OS & 539 & 337 & 0.62 & $0.52-0.74$ & $1.80 \mathrm{E}-07$ \\
\hline & NDUFS8 & 203189_s_at & OS & 588 & 288 & 0.74 & $0.61-0.89$ & 0.0015 \\
\hline & NDUFV1 & 208714_at & OS & 553 & 323 & 1.53 & $1.29-1.82$ & $9.40 \mathrm{E}-07$ \\
\hline & NDUFV2 & 202941_at & OS & 457 & 419 & 0.56 & $0.47-0.67$ & $3.70 \mathrm{E}-11$ \\
\hline
\end{tabular}


NDUFS1

A
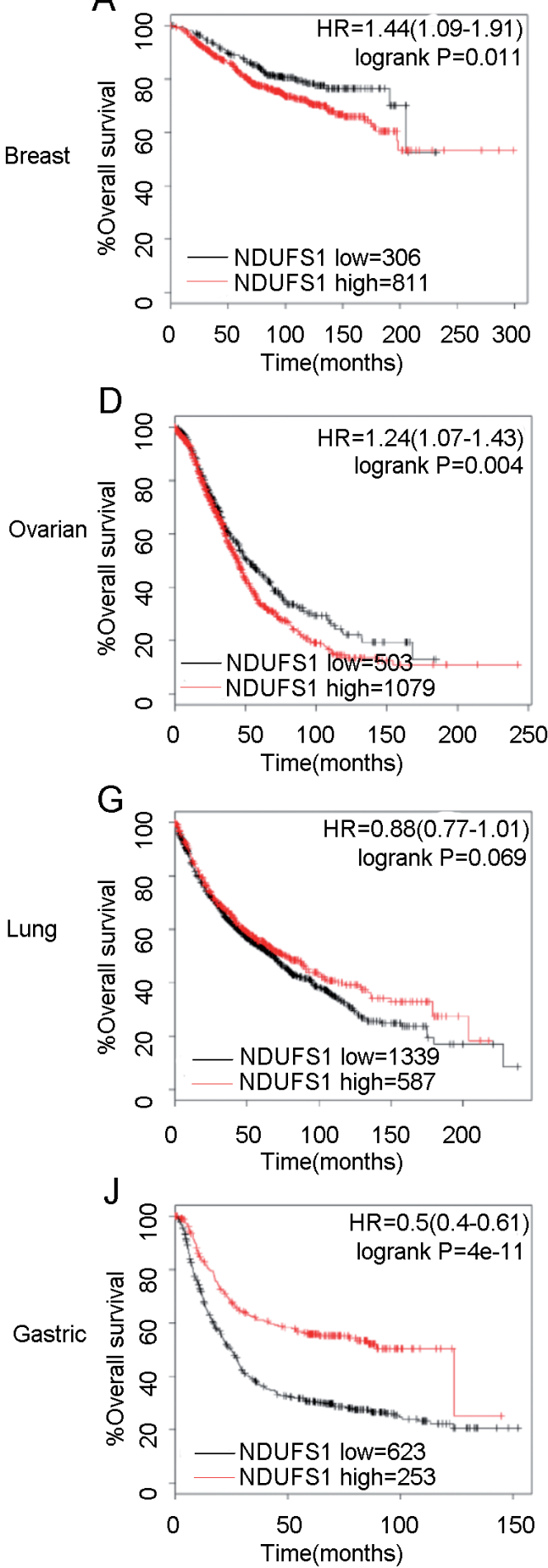

NDUFS2

NDUFS3

B
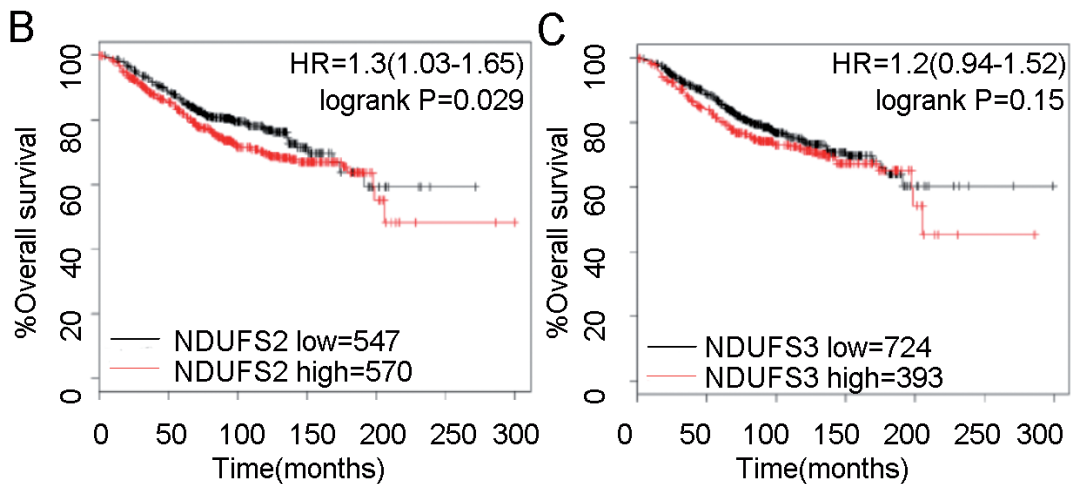

E

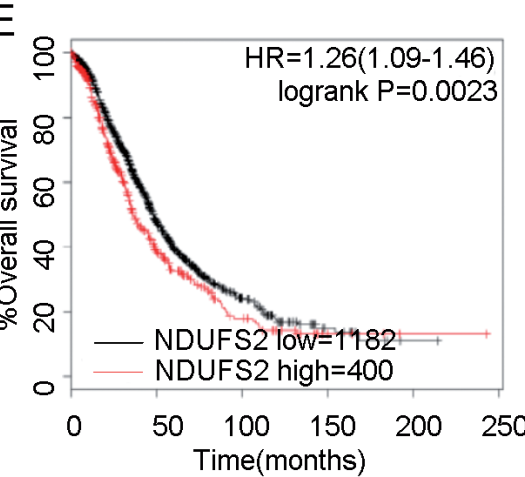

$F$

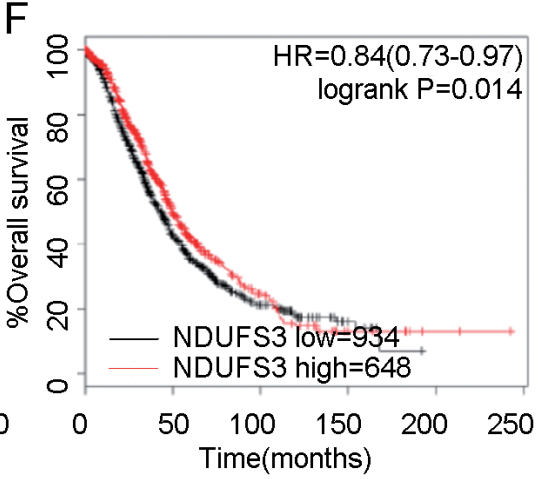

$\mathrm{H}$
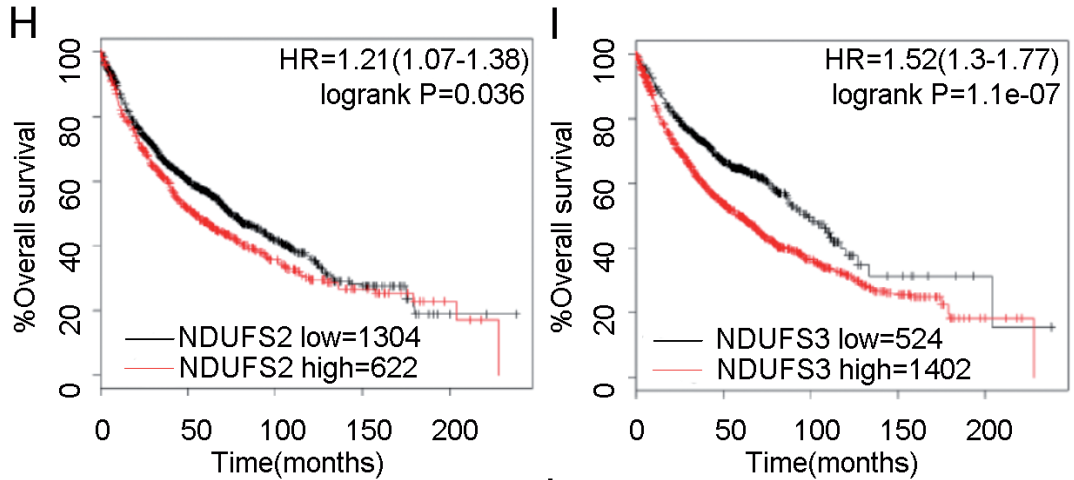

K

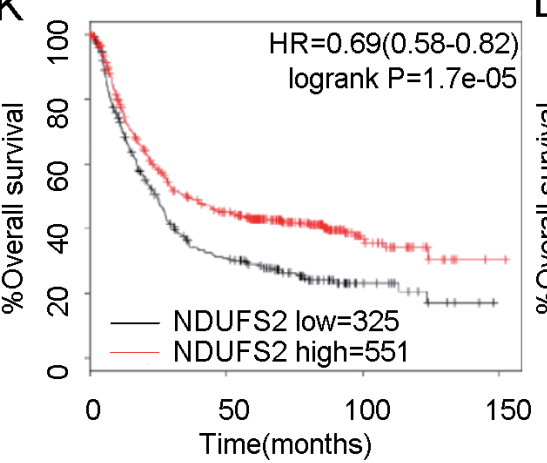

L

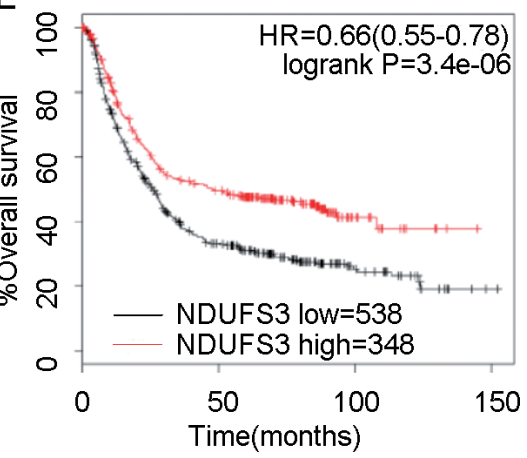

Figure 1. The prognostic value of NDUFS1, NDUFS2, and NDUFS3 expression in four tumor patients

Notes: The desired Affymetrix ID of each gene in each tumor is valid, summarized in Table1; (A-C): Survival curves are plotted for all breast cancer patients ( $n=1117)$. (D-F): Survival curves are plotted for all ovarian cancer patients $(n=1582)$. (G-I): Survival curves are plotted for lung cancer patients $(n=1926)$. (J-L): Survival curves are plotted for gastric cancer patients $(n=876)$. Data was analyzed using Kaplan Meier Plotter (www.kmplot.com). Abbreviation: HR: hazard ratio 
NDUFS7
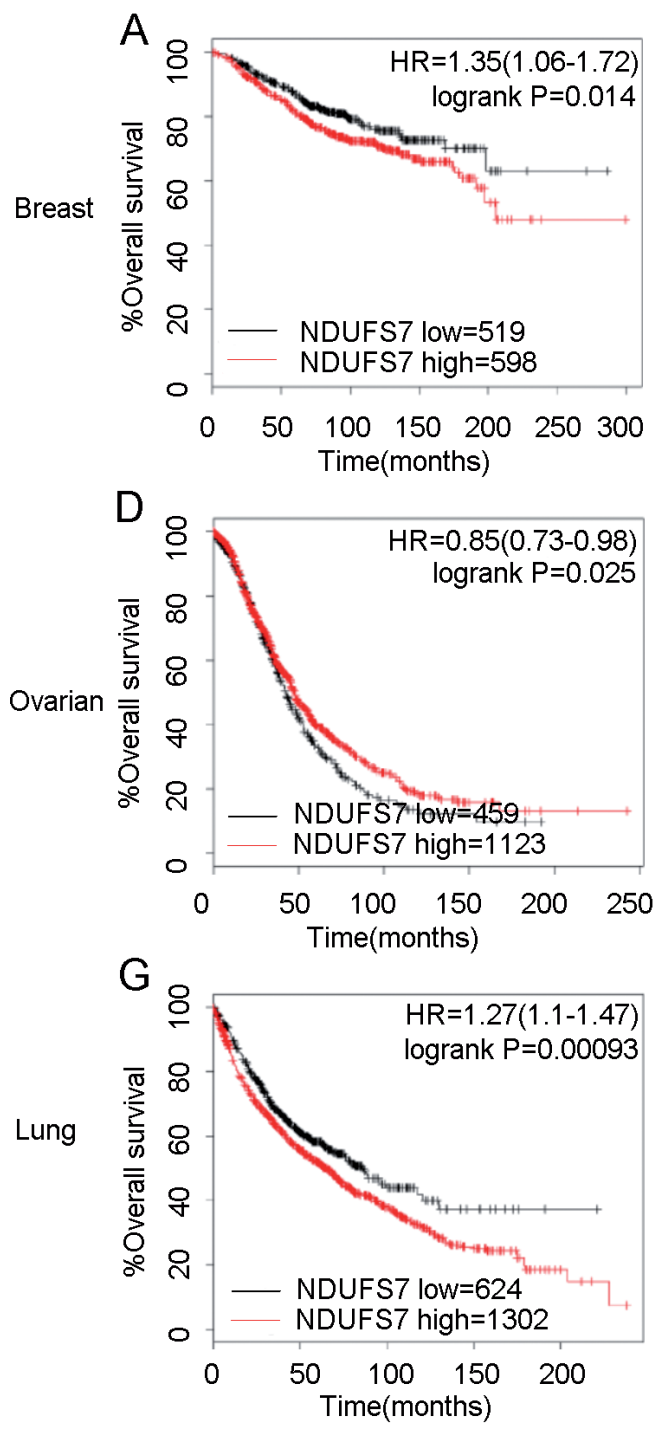

Lung

$\mathrm{J}$

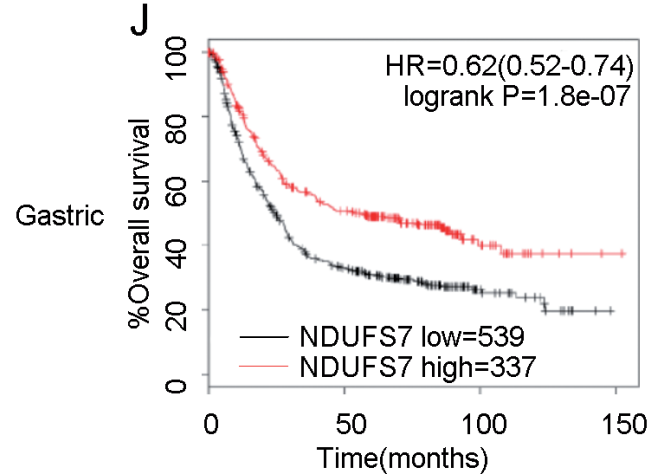

NDUFS8
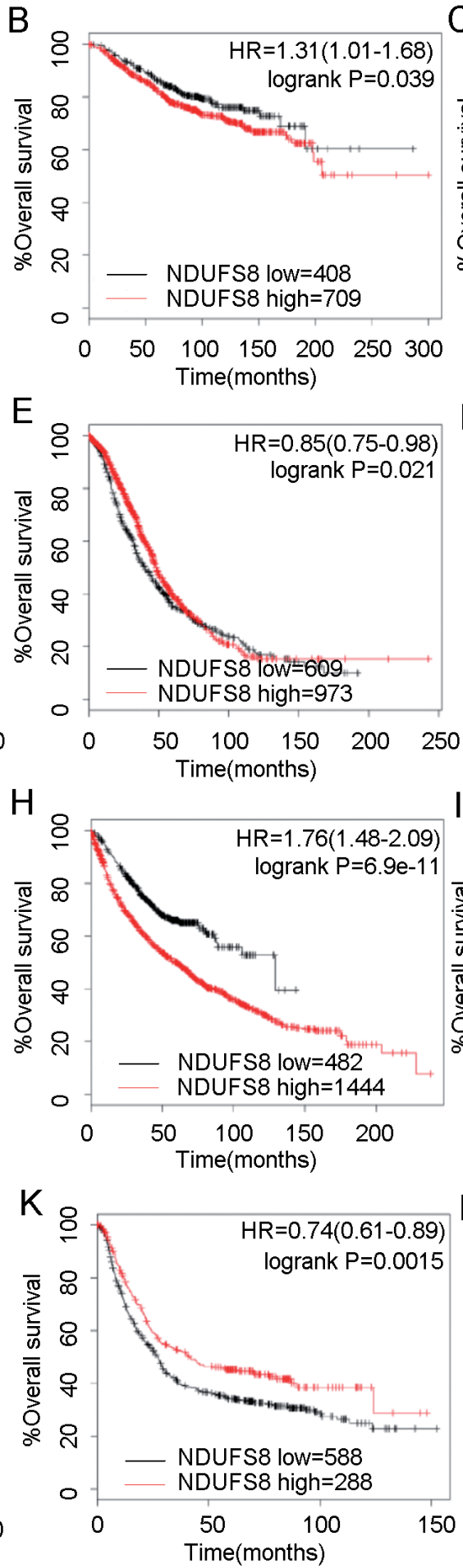

NDUFV1

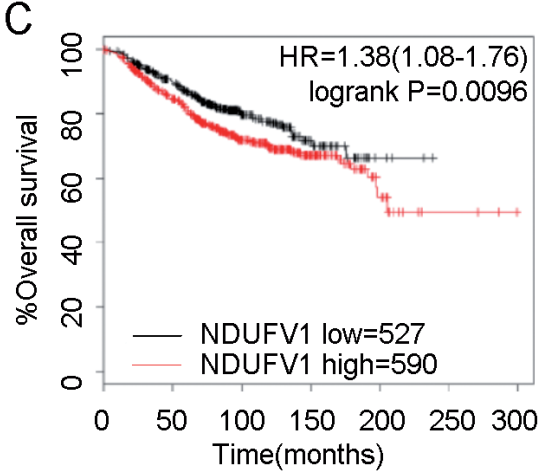

F
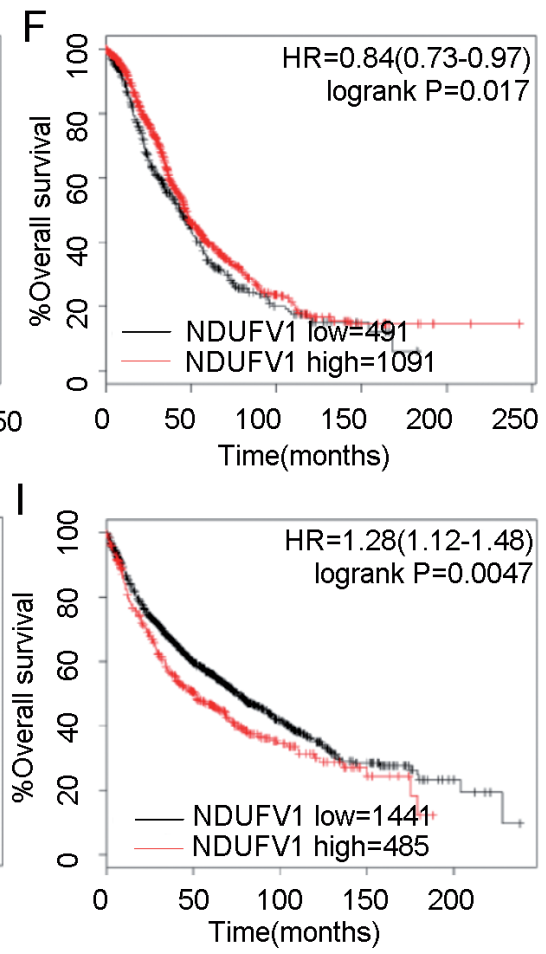

Figure 2. The prognostic value of NDUFS7, NDUFS8, and NDUFV1 expression in four tumor patients

Notes: The desired Affymetrix ID of each gene in each tumor is valid, summarized in Table1; (A-C): Survival curves are plotted for all breast cancer patients $(\mathrm{n}=1117)$. (D-F): Survival curves are plotted for all ovarian cancer patients $(\mathrm{n}=1582)$. (G-I): Survival curves are plotted for lung cancer patients $(\mathbf{n}=1926)$. (J-L): Survival curves are plotted for gastric cancer patients ( $\mathbf{n}=\mathbf{8 7 6})$. Data was analyzed using Kaplan Meier Plotter (www.kmplot.com). Abbreviation: HR: hazard ratio 
For lung cancer, the curves showed that high level of the seven 'core genes' apart from NDUFS1, contributed to worse OS in all lung patients (Figures 1B, 1C, 2A-C, 3C). Although NDUFS1 expression above or below the median separated the patients into two groups, the Log Rank $P$ value, however, was
0.069. Integrated with the other 'all genes' in S-Table4, it could be indicated that 29 out of the 42 'all genes' had worse, and 2 better OS for lung cancer patients (Figure 3E). Additionally, it could be indicated that, except for NDUFS1 predicting better OS, the other members of the "core genes" could predict
A

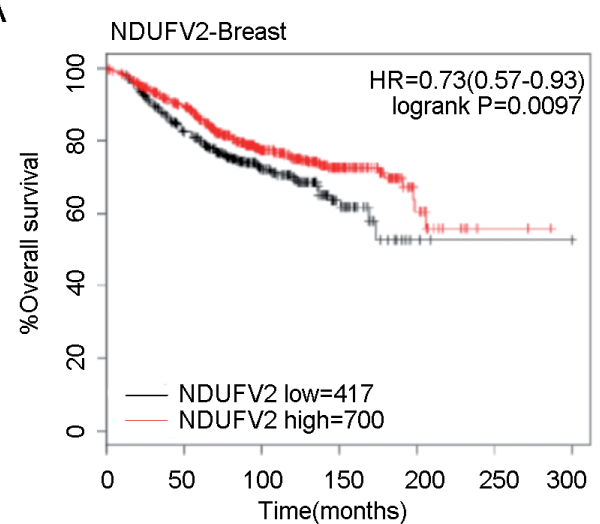

B
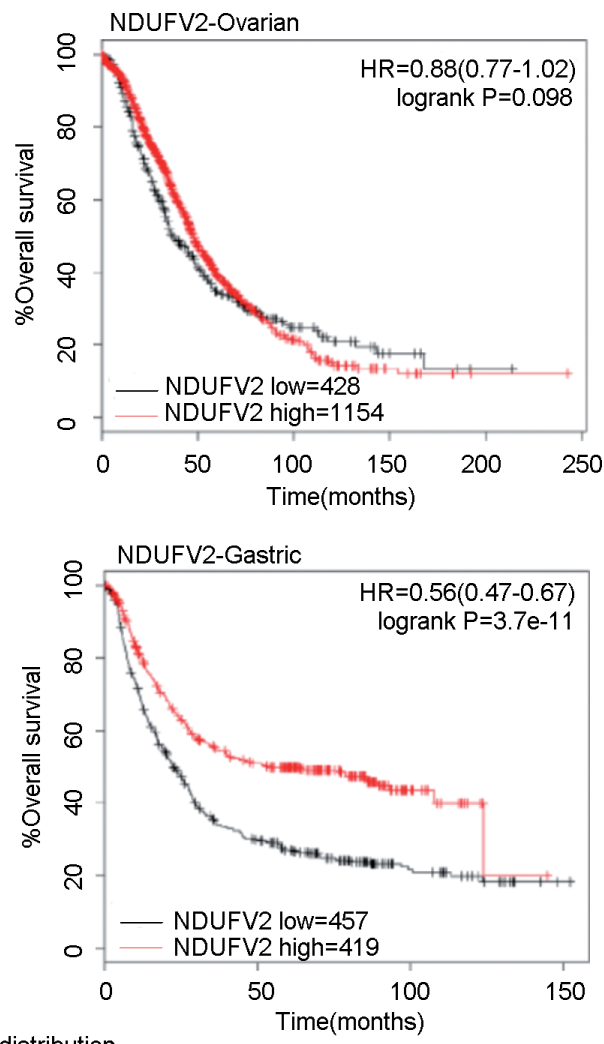

E

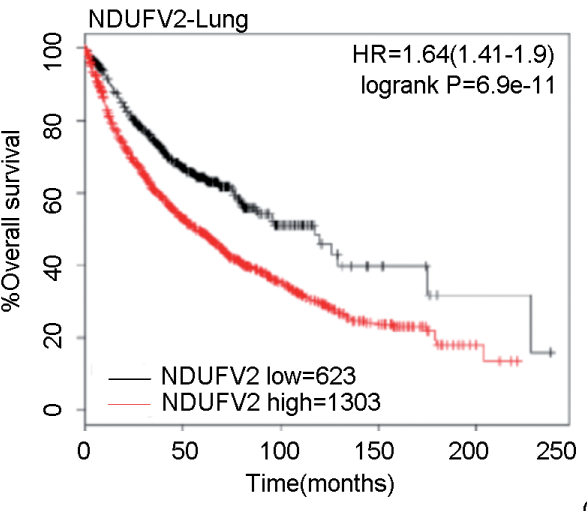

Gene number distribution

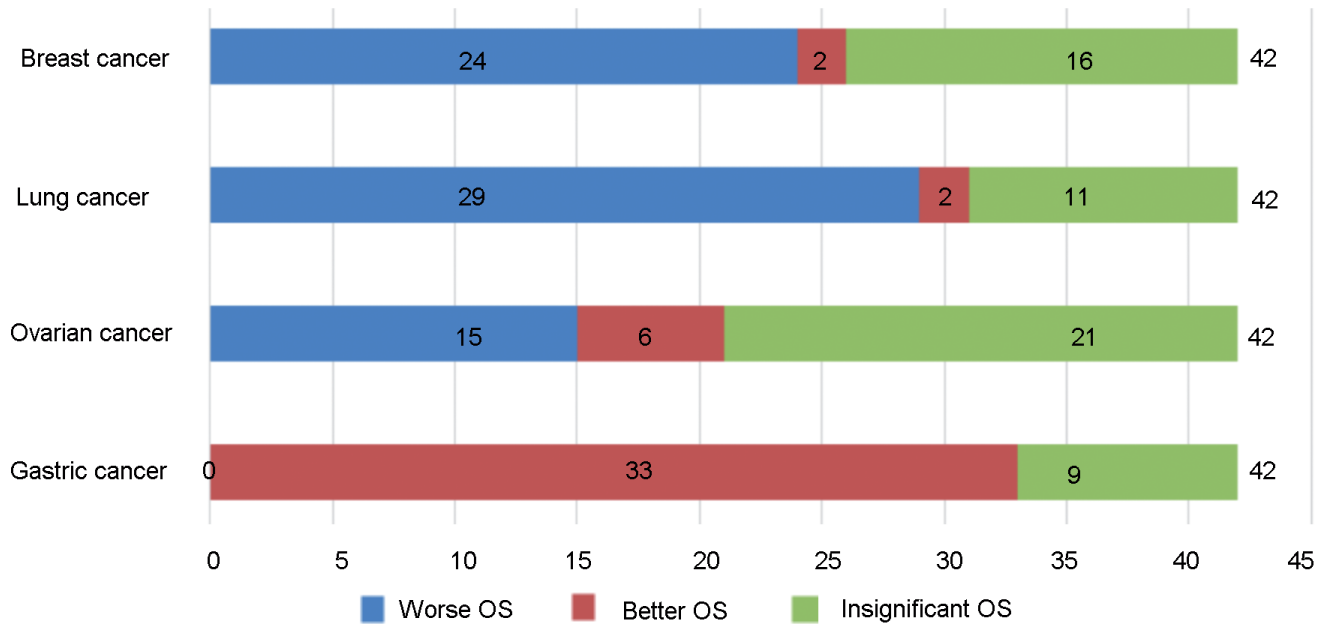

Figure 3. The prognostic value of NDUFV2 expression in four tumor patients and data distribution

Notes: The desired Affymetrix ID of NDUFV2 in each tumor is valid, summarized in Table1; (A-D): Survival curve is plotted for all breast cancer patients $(\mathrm{n}=1117)$, all ovarian cancer patients $(\mathrm{n}=1582)$, for lung cancer patients $(\mathrm{n}=1926)$, and for gastric cancer patients $(\mathrm{n}=\mathbf{8 7 6})$. Data was analyzed using Kaplan Meier Plotter (www.kmplot.com). (E): Gene number distribution of "all genes" in their prognostic effects in different four types of tumors. The desired Affymetrix ID of each gene in each tumor is valid, summarized in S-Table4. Abbreviation: HR: hazard ratio; OS: overall survival. 
worse OS, especially for adenocarcinoma or clinical stage I and II patients, which was in accordance with the NDUFS1 good and others worse for all patients (S-Table3.5 and S-Table3.6). While, whether the patients had smoking history or not, female or male, the "core genes" exhibited similar results as for all patients (S-Table3.7 and S-Table3.8).

What was more meaningful was that, for gastric tumor, the high expression of seven 'core genes', apart from NDUFV1 with worse OS, had identically significant better OS in all gastric cancer patients (Figure 1,2A-C; 3D). Together with the other 'all genes' in S-Table4, we amazingly found that 33 out of the 42 'all genes' had better OS for gastric cancer patients, none with worse OS (Figure 3E). Furthermore, for well differentiated or clinical stage III and IV patients, the "core genes" could predict better OS, except for NDUFV1 predicting worse OS, which was in accordance with the NDUFV1 worse and others good for all patients (S-Table3.9, S-Table3.10). While, whether the patients were intestinal type or diffuse type according to Lauren Classification, female or male, the "core genes" exhibited similar results as for all patients (S-Table3.11, S-Table3.12).

Additionally, we further queried the 'core genes' for other prognostic indicators in the four tumors, including relapse free survival (RFS), post progression survival (PPS) and distant metastasis survival (DMFS) for breast cancer; progression free survival (PFS), and PPS for ovarian cancer; first progression (FP) and PPS for lung cancer; FP for gastric cancer. Survival curves are plotted for all patients (S-Figure 2-5). The desired Affymetrix ID, Cases Number, Hazard Ratio (HR; and 95\% confidence intervals) and Log Rank $P$ were summarized in S-Table5. For breast cancer, as was shown in S-Figure 1, apart from the genes with no prognostic significance (data not shown) and two (NDUFS8 and NDUFV2) with the opposite prognostic effect, the high expression of all the individual "core genes" was correlated with patients' worse survival, in accordance with the effect of them in the OS. For ovarian cancer, as was indicated in S-Figure 2, the majority of the 'core genes' high expression had a good prognostic effect for all patients, only with NDUFS1 the opposite effect on FP. For both lung and gastric cancers, apart from the genes with no prognostic significance (not shown), high expression of the 'core genes' had identical prognostic significance to the OS respectively, as was shown in S-Figure 3 and 4.

Briefly, for overwhelming majority of the 'all genes' with significant OS of the CI, relative expression level of the individual gene had consistent prognostic effect for patients in each of the four tumor separately (Figure 3E).

While from another perspective, it could be demonstrated that for the individual gene, its influence on the four type of different tumor patients' survival were inconsistent, as was indicated in Figure 1 (A, D, G, J) for NDUFS1, Figure 1 (B, E, $\mathrm{H}, \mathrm{K}$ ) for NDUFS2, Figure 1 (C, F, I, L) for NDUFS3, Figure 2 (A, D, G, J) for NDUFS7, Figure 2 (B, E, H, K) for NDUFS8, Figure 2 (C, F, I, L) for NDUFV1, and Figure 3 (A-D) for NDUFV2.
Frequent cumulative alterations of the 'all genes' in various cancers while limited influence in patients' survival. Given that the expression level of individual 'all genes' of the CI had significant and identical influence on patients' survival, then we were interested in investigating the genes' alterations between normal and cancer patients in gene and expression-levels.

From the cross-cancer alteration summary for the 'all genes' in 105 studies including various tumors (Figure 4A, 4B), it could be found that 86 of all the studies exhibited gene changes, among which almost half of them manifested that more than $30 \%$ of cancer patients burdened alterations of the genes in each of the study. Some individual studies even found that more than $80 \%$ patients held abnormal genes. Then we tested the 'core genes' in the same 105 studies. From the cross-cancer alteration summary (Figure 4C, 4D), we found that 26 of the 79 studies showing genetic changes exhibited more than $15 \%$ of patients bearing gene alterations in each study.

Genetic alterations included four types, mutation, deletion, amplification, and multiple alteration, of which the amplification was prominent (Figure 4A, 4C). Additionally, the similar phenomenon occurred in the nuclear genes of the five $\mathrm{CI}$ subcomplexes respectively (http://cbioportal.org).

However, an interesting phenomenon was that, albeit significantly and widely amplification of the 'all genes' and 'core genes' in various tumors, only 5 out of the 86 studies for 'all genes' and 4 out of the 79 studies for 'core genes' exhibited that the cumulative alteration of genes had significant influence on the OS or disease free survival (DFS) of the patients, positive or negative (S-Figure 5A-I).

Furthermore, the alterations of individual gene occurred in only $0-15 \%$ of patients in each of the 105 studies, most of which less than $5 \%$, and rarely was seen their significant influence on patients' survival (http://cbioportal.org).

In conclusion, cumulative or individual alteration of the 'all genes' in gene level could not significantly affect patients survival.

Overexpression of the individual CI subunits' gene was not ubiquitous in tumors versus normal tissues. From the perspective of molecular genetics, amplification is one of many ways in which a gene can be overexpressed [19].

Therefore, we extracted the summary data on transcript expression for the 'core genes' from the database Oncomine for different tumors, focusing on clinical specimens of cancer vs. normal patient datasets.

As was shown in Figure 5, for each of the seven 'core genes', overwhelming majority of the datasets eligible for the screening condition did not show any expression changes. Although limited, the number of overexpression datasets was more than the downregulation number for most genes. Similar results were demonstrated in the other individual gene of the 'all genes' (S-Table6). For gastric and lung tumors, the 'core genes' expression levels were in approximately accordant with their prognostic effects. While for breast and ovarian cancers, some paradoxical phenomena occurred between the 'core 
genes' mRNA levels and their prognostic effects, probably due to some unknown mechanisms, which remained to be elucidated.

Thus we could concluded that it was not ubiquitous for the overexpression of the individual CI subunits' gene in tumors versus normal tissues.

\section{Discussion}

As an emerging hallmark of tumor, [20] reprogrammed energy metabolism can be caused by aberration of oncogene and tumor suppressor genes, [21] as well as can result from mutations in mtDNA [4]. Consequently OXPHOS function is disturbed, and ROS production elevated, then DNA damaged and oncogenic signaling pathways activated, which can further promote cancer cell metabolic reprogramming, hypoxia adaptation, uncontrolled growth and metastasis [3]. A vicious circle forms.

Eighty years ago, Warburg observed that tumor cells produced excess lactate in the presence of oxygen due to mitochondrial dysfunction, [22] which was known as the "Warburg effect' or aerobic glycolysis. However, it is now clear that many tumor cells are capable of performing oxidative phosphorylation, and mitochondrial function is essential for cancer cell viability [23]. The 'reverse Warburg effect' presents that ROS produced from cancer cells can cause adjacent stromal fibroblasts cells mitophagy through inactivating caveolin-1, then lactate production increases in these stromal cells, which can further fuel cancer cell oxidative metabolism [24].

During tumor progression, cancer cells suffer various survival pressures, including the insufficient in nutrient and $\mathrm{O}_{2}$ supplying, and the survivors must undergo processes
A Genes of Complex I All Subunits

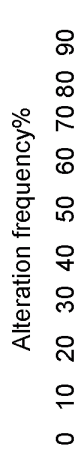

B

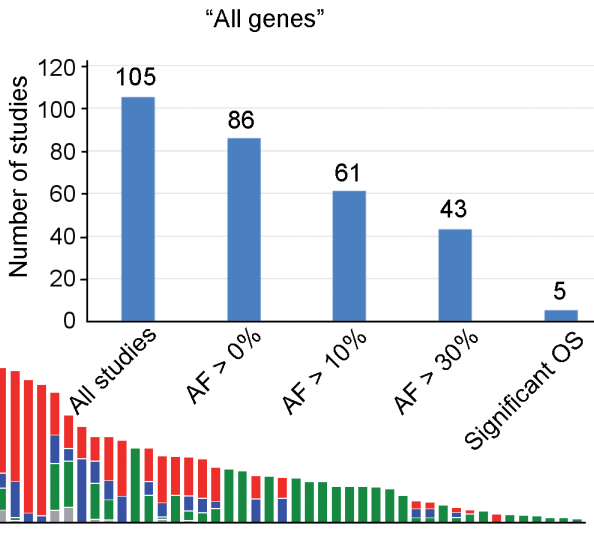

86 of the 105 studies from the cBio Portal*

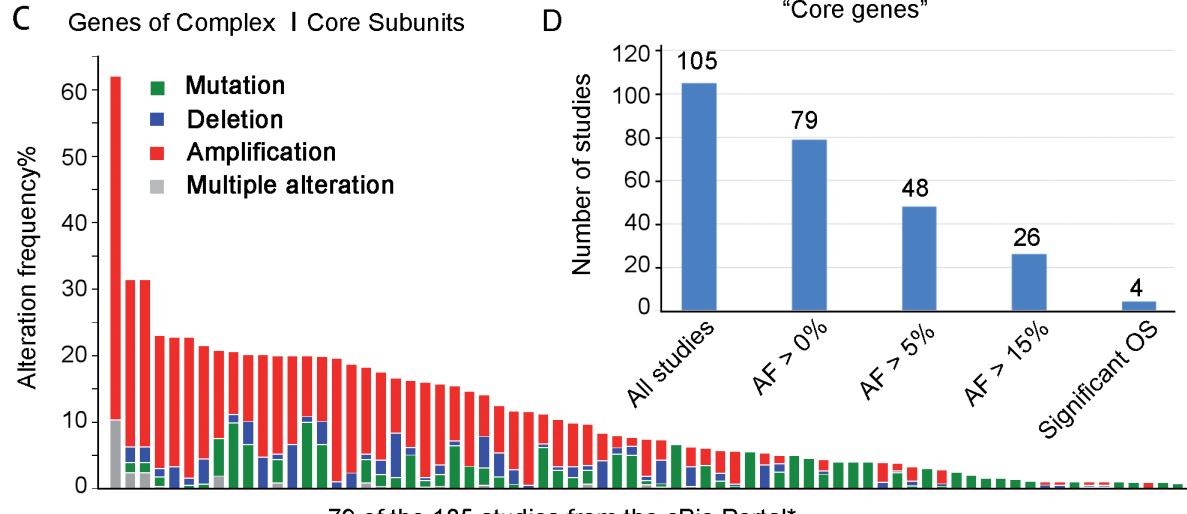

79 of the 105 studies from the cBio Portal ${ }^{*}$

Figure 4. Cross-cancer alteration summary for the 42 "all genes" and 7 "core genes" in 105 studies.

Notes: ${ }^{\star}$ Order of the studies was seen in S-Table7; the alterations include mutation, deletion, amplification, and multiple alteration. (A): Cumulative alteration frequency of the "all genes" for each study was indicated. (B): Study number distribution of cumulative alteration frequency of the "all genes". (C): Cumulative alteration frequency of the "core genes" for each study was indicated. Data was analyzed using the cBio Cancer Genomics Portal (http:// cbioportal.org). (D): Study number distribution of cumulative alteration frequency of the "core genes". Abbreviation: HR: hazard ratio; MSKCC: memorial sloan-kettering cancer center; TCGA: the cancer genome atlas; ICGC: international cancer genome consortium; AF: alteration frequency. 

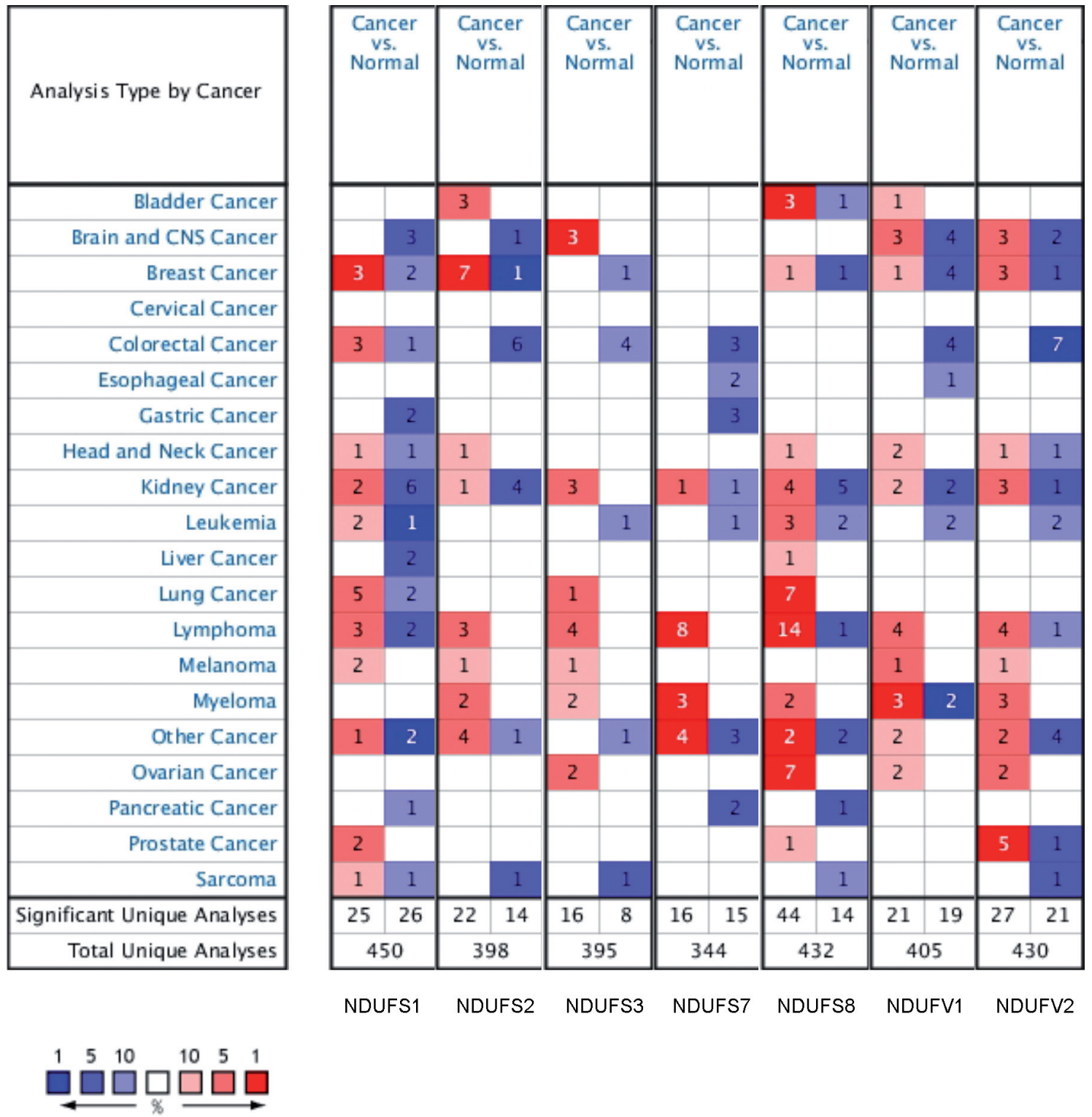

Cell color is determined by the best gene rank percentile for the analyses within the cell.

NOTE: An analysis may be counted in more than one cancer type.

Figure 5. The "core genes" mRNA expression in different tumor types from Oncomine Notes: This graphic compares the number of datasets that had significant mRNA overexpression (left column, red) and underexpression (right column, blue) of the specified gene in cancer versus normal tissue. The datasets were obtained with the following parameters: $P$ value threshold of 0.05 , more than 1.5 fold change and top $10 \%$ of the gene rank.

of metabolic reprogramming and hypoxic adaptation [25]. In cancer biology, a still open question concerns whether metabolic genes including those encoding CI subunits play the oncogenic or oncosuppressor role [26].

As the first and crucial component of mitochondrial respiratory chain, CI plays a crucial role in several biochemical processes, such as the cellular redox status $\left(\mathrm{NAD}^{+} / \mathrm{NADH}\right.$ ratio and ROS levels) maintenance, mitochondrial membrane potential generation and finally for ATP production [27].
Moreover, for highly proliferating tumor cells, mitochondrial respiratory chain function is indispensable for the synthesis of the pyrimidines that cells used to divide [28]. Thus, disturbance in CI stability or activity not only undermine mitochondrial functions but also destruct cellular homeostasis, inducing widespread consequences such as activation of autophagy or apoptosis [29].

It seems that complete disassembly of CI caused by nucleotide alterations is disadvantageous for the tumor, i.e. 
leading to HIF1a destabilization, with adverse effect on hypoxic adaptation [30]. Conversely, those less destructive somatic mutations, such as missense variants, may be maintained in tumor tissue, as they may instead positively promote HIF $1 \alpha$ function and contribute to metabolic adaptation through decreasing, but not abrogating, CI function [30].

Oxygenation is actually not necessarily static in tumor cells but instead waves regionally and temporally, ranging from normoxia to hypoxia, due to the instability and chaotic organization of the tumor-associated neovasculature, [31] which exposes the mitochondria in variable oxygen conditions. Thus together with the "reverse Warburg effect", in which two subpopulations of cancer cells function symbiotically to fuel tumor growth, we can conclude that the OXPHOS may functions unstably in different tumors or in different stages or regions of one tumor. Additionally, it is well demonstrated that ROS plays a dose-dependent role in promoting cancer cells surviving or dying [32]. From the above, it is not difficult to understand the result that the relative expression level of the CI 'all genes' had reverse prognosis effect in different cancers, although the exact mechanisms are still to be elucidated. Due to tumor heterogeneity, the CI genes function might also vary, or even antipodal in the different subtypes or different stages of the same type of tumor. Thus precision medicine and individualized treatment were to be taken into account in their future clinical application.

Although the cumulative alterations of 'all genes' accounted for a large proportion in the thousands of patients populations, however, once specific to each person, maybe there were only partial or even individual gene alterations. Additionally, due to the diverse alterations, including mutation, deletion, amplification, and multiple alteration, it was hard to evaluate how the single amplification affect patients' survival.

Being programmed by proliferation-inducing oncogenes, reprogrammed energy metabolism was viewed as an emerging hallmark of tumor [20]. Thus it was proposed that function of the multi-subunits comprising CI might not be affected by only partial or individual subunits alterations, but the 'all genes' might be up or down regulated simultaneously in response to the respiratory chain activity. And the overall expression level and their threshold for survival evaluation of CI subunits for tumor patients were expected for investigation in large scale of population through the same platform by the uniform standards.

Although data from the three independent platforms were not comparative, while, all the three platforms contained as much as thousands of patients' information, to some extent, at least, they were illustrative enough to suggest the conclusions. And further investigation with more perfect design was still needed.

In this study, we presented that for overwhelming majority of the 'all genes' of the CI, relative expression level of the individual gene had consistent prognostic effect for patients in breast, ovarian, lung, and gastric tumors separately. However frequent cumulative or individual alterations of the 'all genes' in gene level could not significantly affect patients' survival. And the overexpression of the individual CI subunits' gene was not ubiquitous in tumors versus normal tissues. Given that reprogrammed energy metabolism was viewed as an emerging hallmark of tumor, thus tumor patients' survival might potentially to be evaluated by the overall expression level of CI subunits.

Comprehensive understanding of the nuclear genome encoded CI subunits may have guiding significance for the diagnosis and prognosis in tumor patients. Based on our study, the discovery of the systematic molecular mechanisms that how CI subunits reflect or lead to different outcomes of tumor patients can pave a way for more effective tumor diagnosis and treatment.

Acknowledgements: This work was supported by the grants from National Natural Science Foundation of China (81272923 and 81302299), Program for New Century Excellent Talents in University (NCET-12-0127).

Supplementary information is available in the online version of the paper.

Notes: All of the supplementary information (S-Tables and S-Figures) legends are indicated in S-legends. S-Table3.1-3.12 were indicated in S-Table3 file, and the other S-Tables were in the S-Tables file.

\section{References}

[1] SIEGEL R. L, MILLER K. D, JEMAL A. Cancer statistics, 2015. CA Cancer J Clin. (c) 2015 American Cancer Society. 2015; 1: 5-29.

[2] CHAFFER C. L, WEINBERG RAA. Perspective on cancer cell metastasis. Science. 2011; 6024: 1559-64. http://dx.doi. org/10.1126/science. 1203543

[3] IOMMARINI L, CALVARUSO MA, KURELAC I, GASPARRE G, PORCELLI AM. Complex I impairment in mitochondrial diseases and cancer: parallel roads leading to different outcomes. Int J Biochem Cell Biol. Copyright (c) 2012 Elsevier Ltd. All rights reserved. 2013; 1: 47-63.

[4] LU J, SHARMA LK, BAI Y. Implications of mitochondrial DNA mutations and mitochondrial dysfunction in tumorigenesis. Cell Res. 2009; 7: 802-15. http://dx.doi.org/10.1038/ cr.2009.69

[5] BRANDON M. and BALDI P. and WALLACE, D. C. Mitochondrial mutations in cancer. Oncogene. 2006; 34: 4647-62. http://dx.doi.org/10.1038/sj.onc.1209607

[6] SCHON EA, DIMAURO S, HIRANO M. Human mitochondrial DNA: roles of inherited and somatic mutations. Nat Rev Genet. 2012; 12: 878-90. http://dx.doi.org/10.1038/ $\underline{\text { nrg } 3275}$

[7] GASPARRE G, KURELAC I, CAPRISTO M, IOMMARINI L, and GHELLI A, et al. A mutation threshold distinguishes the antitumorigenic effects of the mitochondrial gene MTND1, 
an oncogenes function. Cancer Res. 2011; 19: 6220-29. http:// dx.doi.org/10.1158/0008-5472.CAN-11-1042

[8] PARK JS, SHARMA LK, LI H, XIANG R, HOLSTEIN D, et al. A heteroplasmic, not homoplasmic, mitochondrial DNA mutation promotes tumorigenesis via alteration in reactive oxygen species generation and apoptosis. Hum Mol Genet. 2009; 9: 1578-89. http://dx.doi.org/10.1093/hmg/ddp069

[9] PELICANO H, XU RH, DU M, FENG L, SASAKI R, et al. Mitochondrial respiration defects in cancer cells cause activation of Akt survival pathway through a redox-mediated mechanism. J Cell Biol. 2006; 6: 913-23. http://dx.doi.org/10.1083/ jcb. 200512100

[10] HIRST J. Mitochondrial complex I. Annu Rev Biochem. 2013; 551-75. http://dx.doi.org/10.1146/annurev-biochem-070511103700

[11] CHEN R, FEARNLEY IM, PEAK-CHEW SY, WALKER JE. The phosphorylation of subunits of complex I from bovine heart mitochondria. J Biol Chem. 2004; 25: 26036-45. http:// dx.doi.org/10.1074/jbc.M402710200

[12] HIRST J. Why does mitochondrial complex I have so many subunits? Biochem J. 2011;2: e1-03. http://dx.doi.org/10.1042/ $\underline{\text { BJ20110918 }}$

[13] MIHALY Z, KORMOS M, LANCZKY A, DANK M, BUDCZIES J, et al. A meta-analysis of gene expression-based biomarkers predicting outcome after tamoxifen treatment in breast cancer. Breast Cancer Res Treat. 2013;2: 219-32. http:// dx.doi.org/10.1007/s10549-013-2622-y

[14] GYORFFY B, LANCZKY A, EKLUND AC, et al. An online survival analysis tool to rapidly assess the effect of 22,277 genes on breast cancer prognosis using microarray data of 1,809 patients. Breast Cancer Res Treat. 2010; 3: 725-31. http:// dx.doi.org/10.1007/s10549-009-0674-9

[15] GYORFFY B, LANCZKY A, SZALLASI Z. Implementing an online tool for genome-wide validation of survival-associated biomarkers in ovarian-cancer using microarray data from 1287 patients. Endocr Relat Cancer. 2012; 2: 197-208. http:// dx.doi.org/10.1530/ERC-11-0329

[16] GYORFFY B, SUROWIAK P, BUDCZIES J, LANCZKY A. Online survival analysis software to assess the prognostic value of biomarkers using transcriptomic data in non-smallcell lung cancer. PLoS One. 2013; 12: e82241. http://dx.doi. org/10.1371/journal.pone.0082241

[17] CERAMI E, GAO J, DOGRUSOZ U, GROSS BE, SUMER SO, et al. The cBio cancer genomics portal: an open platform for exploring multidimensional cancer genomics data. Cancer Discov. (c) 2012 AACR., 2012; 5: 401-04.

[18] RHODES DR, YU J, SHANKER K, DESHPANDE N, VARAMBALLY, R, et al. ONCOMINE: a cancer microarray database and integrated data-mining platform. Neoplasia. 2004; 1: 1-06. http://dx.doi.org/10.1016/S1476-5586(04)80047-2

[19] MYLLYKANGAS S, KNUUTILA S, MANIFESTATION, MECHANISMS AND MYSTERIES OF GENE AMPLIFICATIONS. Cancer Lett. 2006; 1: 79-89. http://dx.doi. org/10.1016/j.canlet.2005.07.045
[20] HANAHAN D, WEINBERG RA, Hallmarks of cancer: the next generation. Cell. Copyright (c) 2011 Elsevier Inc. All rights reserved. 2011; 5: 646-74.

[21] KOPPENOL WH, BOUNDS PL, DANG CV, Otto Warburg's contributions to current concepts of cancer metabolism. Nat Rev Cancer. 2011; 5: 325-37. http://dx.doi.org/10.1038/nrc3038

[22] WARBURG O, On the origin of cancer cells. Science. 1956; 3191: 309-14. http://dx.doi.org/10.1126/ science.123.3191.309

[23] FREZZA C, GOTTLIEB E, Mitochondria in cancer: not just innocent bystanders. Semin Cancer Biol. 2009; 1:4-11. http:// dx.doi.org/10.1016/j.semcancer.2008.11.008

[24] BONUCCELLI G, WHITAKER-MENEZES D, CASTELLOCROS R, PAVLIDES S, PESTELL RG, et al. The reverse Warburg effect: glycolysis inhibitors prevent the tumor promoting effects of caveolin-1 deficient cancer associated fibroblasts. Cell Cycle. 2010; 10: 1960-71. http://dx.doi. org/10.4161/cc.9.10.11601

[25] BERETTA S, WOOD JP, DERHAM B, SALA G, and TREMOLIZZO L, et al. Partial mitochondrial complex I inhibition induces oxidative damage and perturbs glutamate transport in primary retinal cultures. Relevance to Leber Hereditary Optic Neuropathy (LHON). Neurobiol Dis. 2006; 2: 308-17. http://dx.doi.org/10.1016/j.nbd.2006.07.016

[26] CAIRNS RA, HARRIS IS, MAK TW, Regulation of cancer cell metabolism. Nat Rev Cancer. 2011; 2: 85-95. http://dx.doi. org/10.1038/nrc2981

[27] ROESTENBERG P, MANJERI GR, VALSECCHI F, SMEITINK JA, WILLEMS PH, et al. Pharmacological targeting of mitochondrial complex I deficiency: the cellular level and beyond. Mitochondrion. Copyright (c) 2011 Elsevier B.V. and Mitochondria Research Society. All rights reserved. 2012; 1: 57-65.

[28] KING MP, KOGA Y, DAVIDSON M, SCHON EA, Defects in mitochondrial protein synthesis and respiratory chain activity segregate with the tRNA(Leu(UUR)) mutation associated with mitochondrial myopathy, encephalopathy, lactic acidosis, and strokelike episodes. Mol Cell Biol. 1992; 2: 480-90. http:// dx.doi.org/10.1128/MCB.12.2.480

[29] THOMPSON CB, Metabolic enzymes as oncogenes or tumor suppressors. N Engl J Med. 2009; 8: 813-15. http://dx.doi. org/10.1056/NEJMe0810213

[30] PORCELLI AM, ANGELIN A, GHELLI A, MARIANI E, MARTINUZZI A, et al. Respiratory complex I dysfunction due to mitochondrial DNA mutations shifts the voltage threshold for opening of the permeability transition pore toward resting levels. J Biol Chem. 2009; 4: 2045-52. http:// dx.doi.org/10.1074/jbc.M807321200

[31] HARDEE ME, DEWHIRST MW, AGARWAL N, SORG BS, Novel imaging provides new insights into mechanisms of oxygen transport in tumors. Curr Mol Med. 2009; 4: 435-41. http://dx.doi.org/10.2174/156652409788167122

[32] LIOU GY, STORZ P, Reactive oxygen species in cancer. Free Radic Res. 2010; 5: 479-96. http://dx.doi. org/10.3109/10715761003667554 
A.
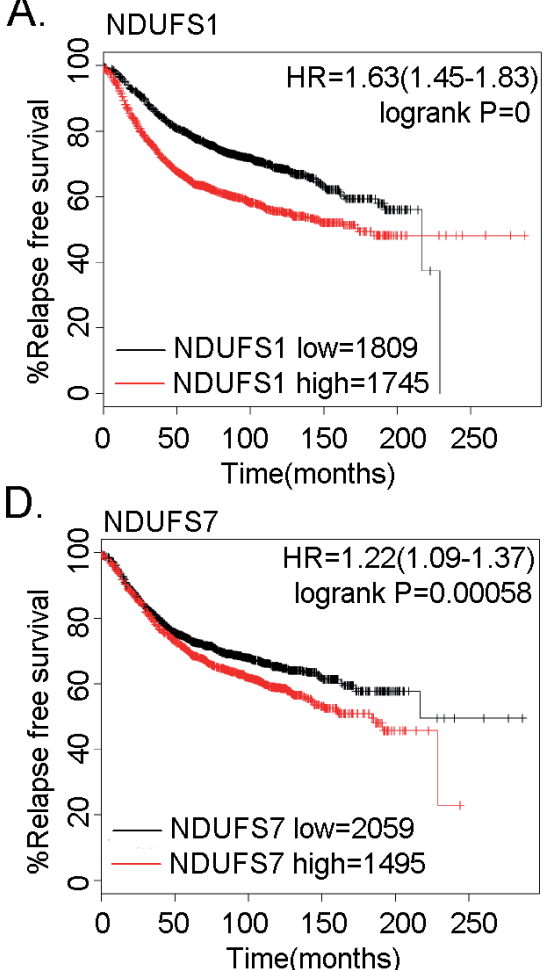

G. NDUFV1

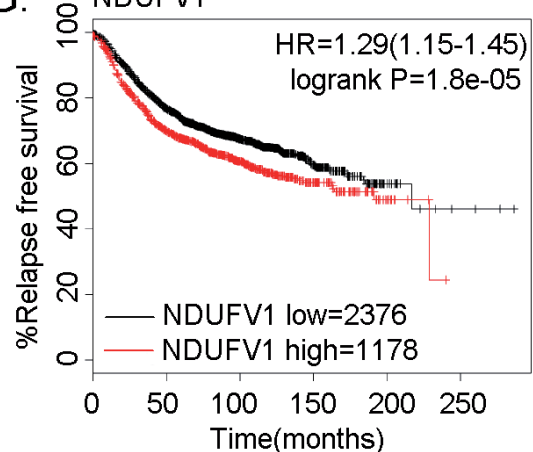

J.

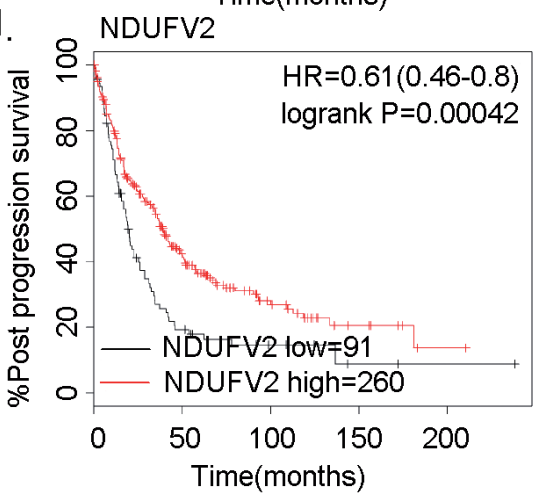

B.

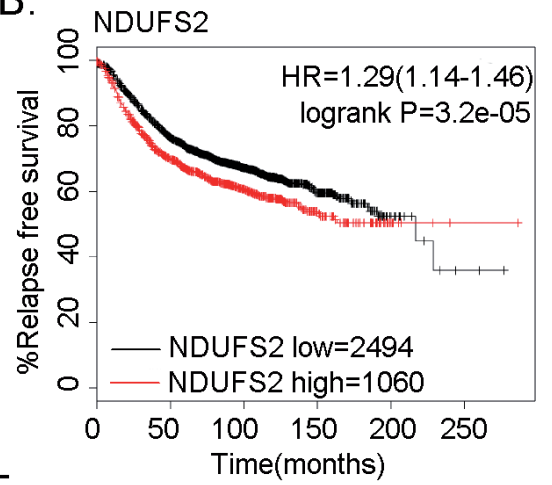

E.

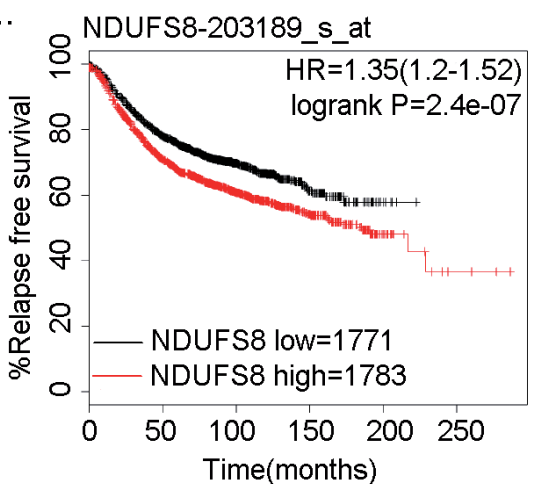

H.

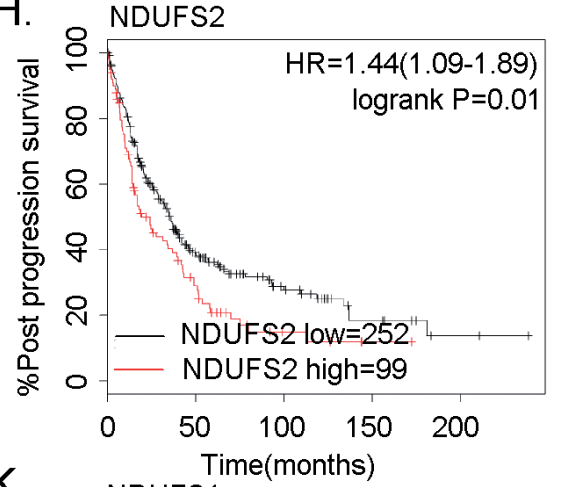

K.

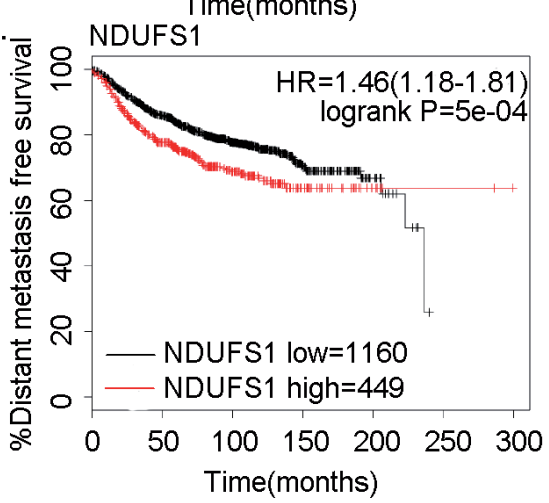

C.

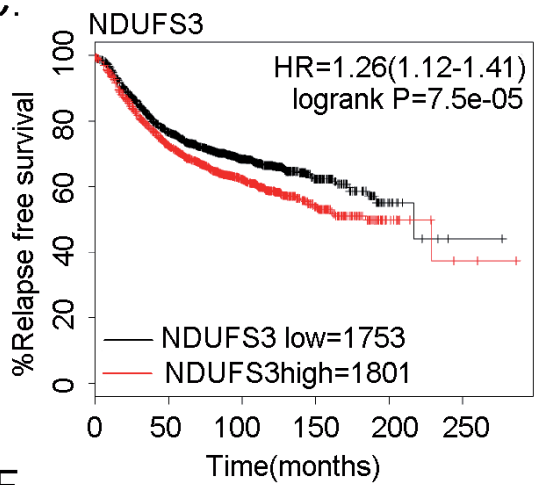

F.

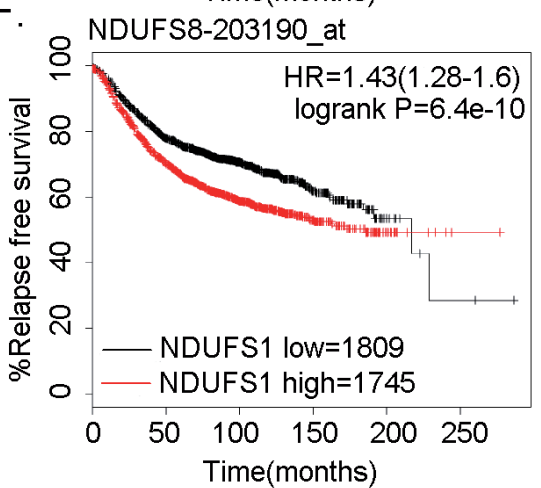

I.

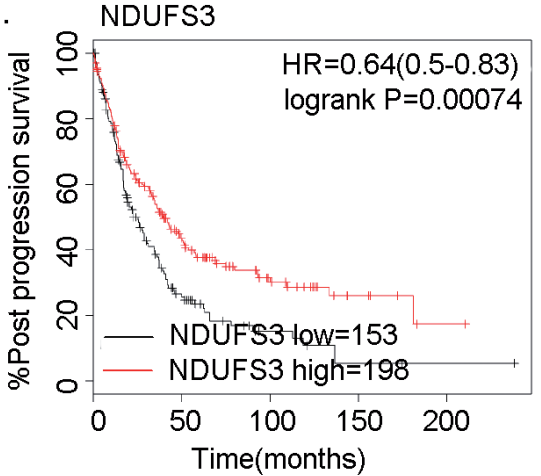

L.

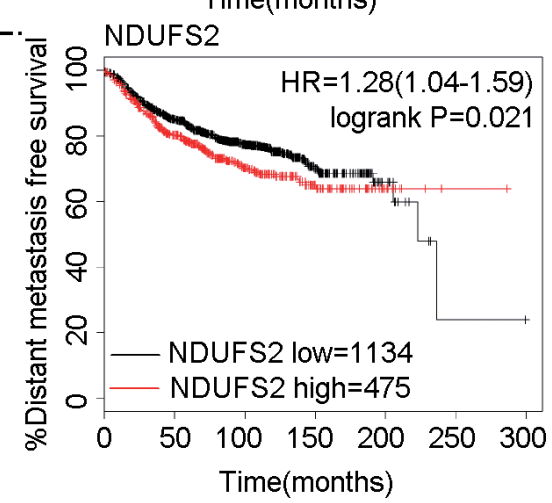

S-Figure 1. The prognostic value of the "core genes" expression in breast cancer patients

Notes: The desired Affymetrix ID of each gene in each tumor is valid, summarized in S-Table5. (a-1): Survival curves are plotted for all breast cancer patients of RFS ( $n=3554)$, DMFS $(n=1609)$ and PPS $(n=351)$. Data was analyzed using Kaplan Meier plotter (www.kmplot.com).

Abbreviation: HR: hazard ratio; RFS: relapse free survival; DMFS: distant metastasis survival; PPS: post progression survival. 
A.

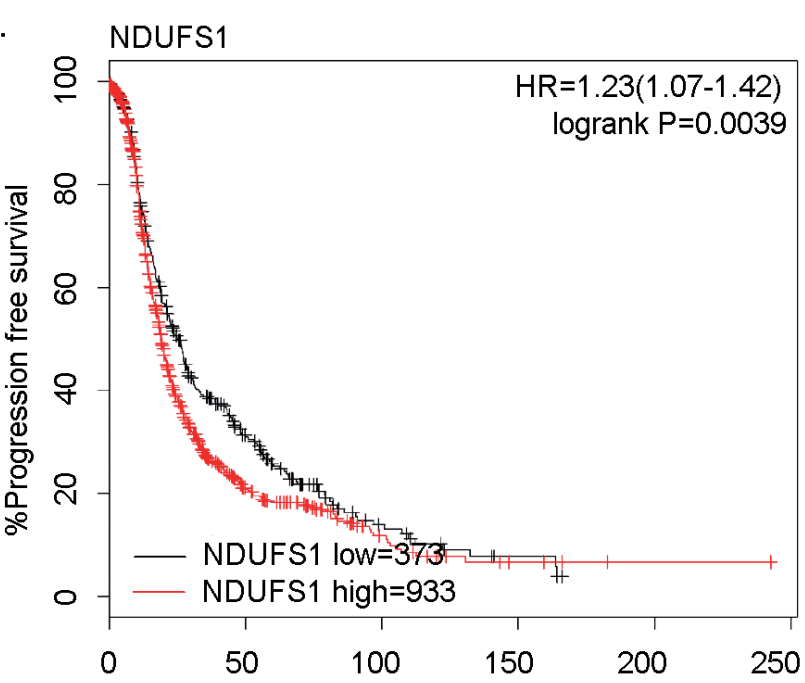

C.

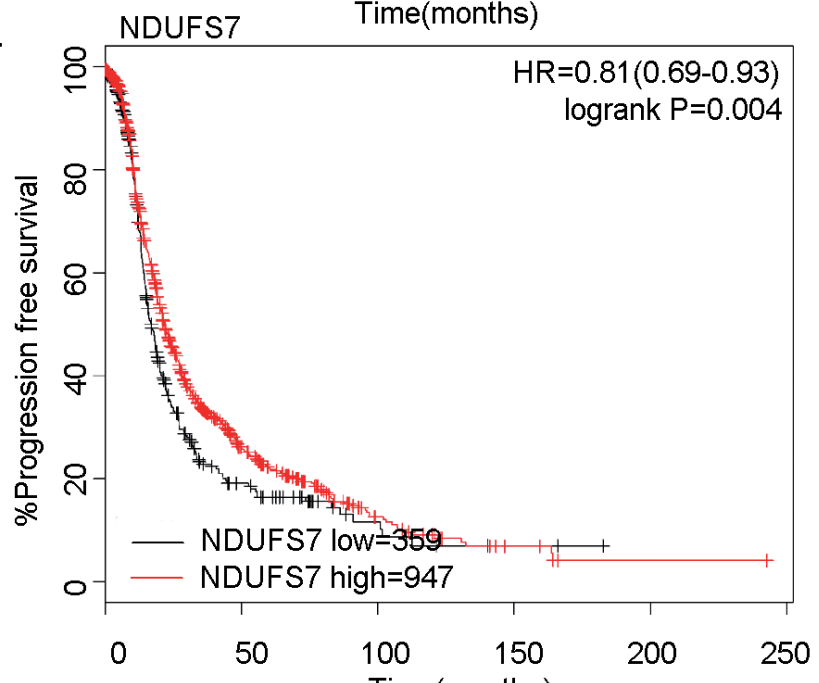

E. NDUFS8 Time(months)

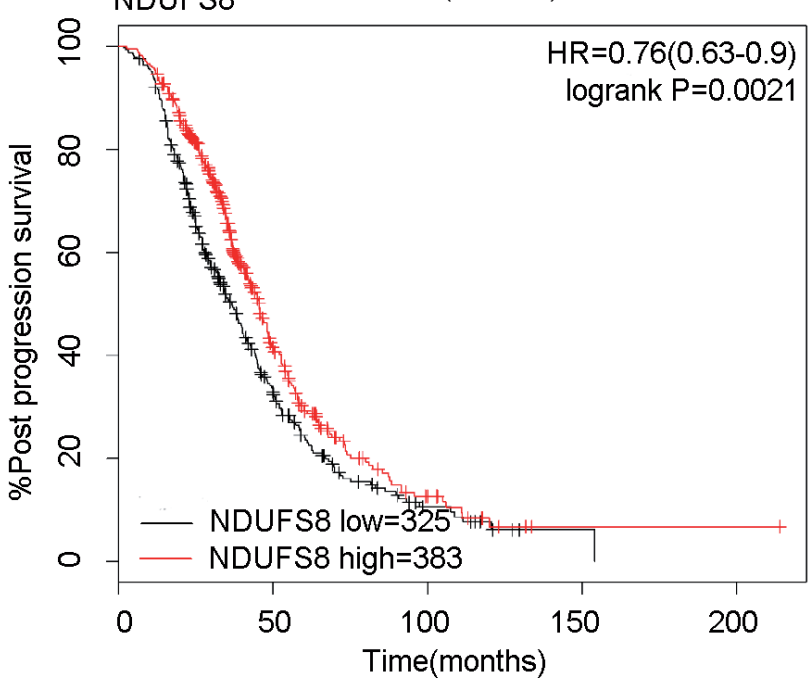

B.

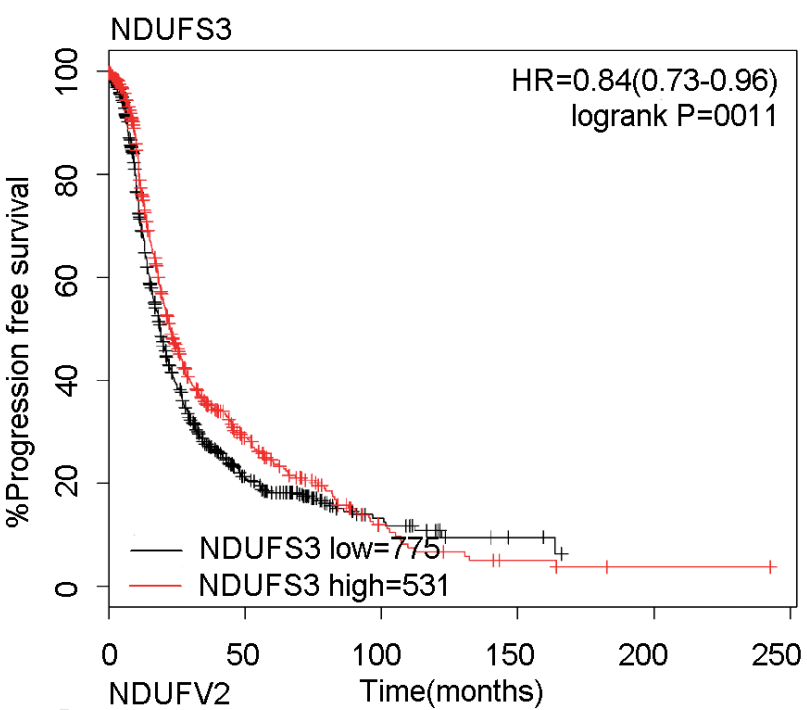

D.

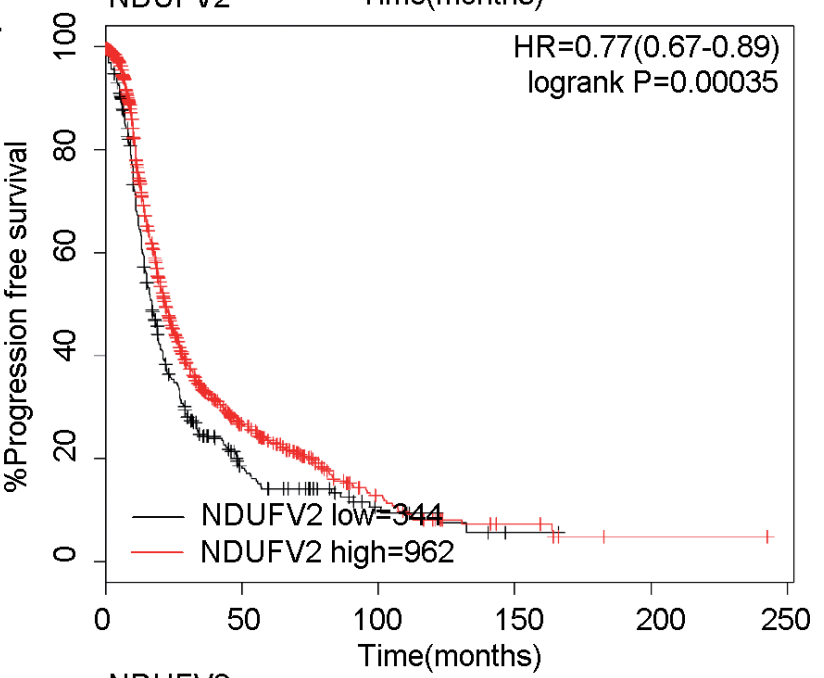

F.

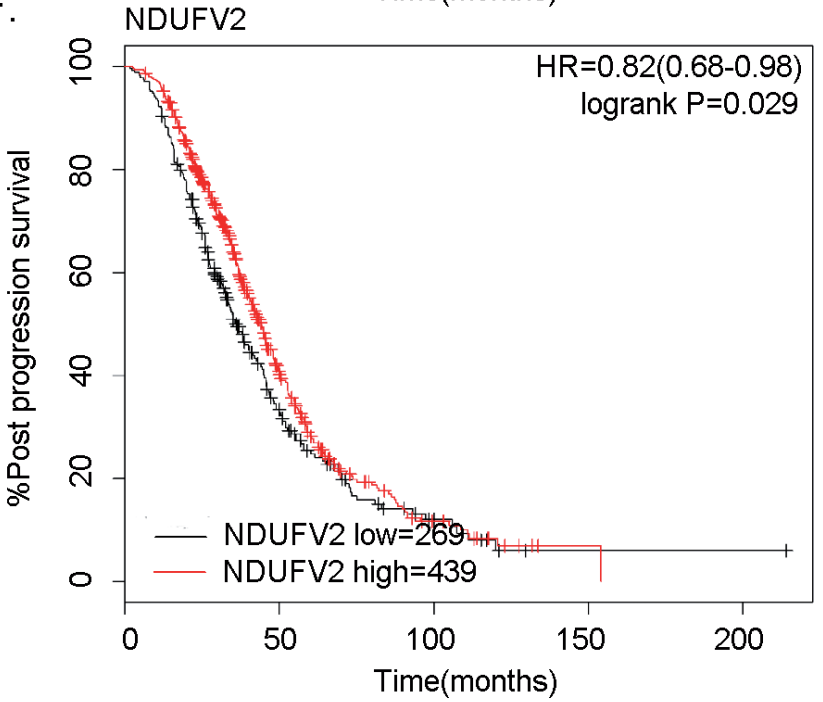

S-Figure 2. The prognostic value of the "core genes" expression in ovarian cancer patients

Notes: The desired Affymetrix ID of each gene in each tumor is valid, summarized in S-Table5. (a-f): Survival curves are plotted for all ovarian cancer patients of PFS $(n=1306)$, and PPS $(n=708)$. Data was analyzed using Kaplan Meier plotter (www.kmplot.com).

Abbreviation: HR: hazard ratio; PFS: progression free survival; PPS: post progression survival. 
A.

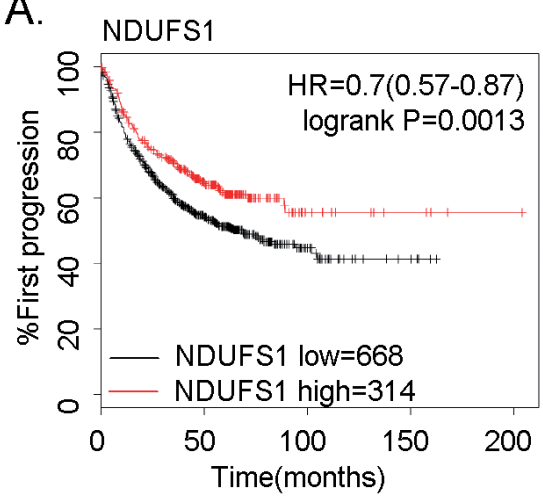

D.
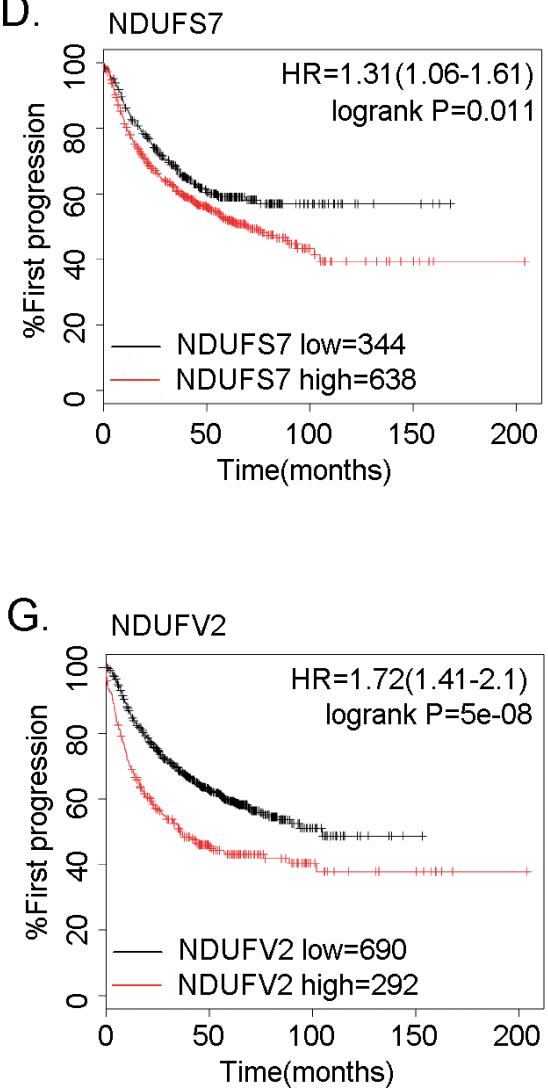

B.

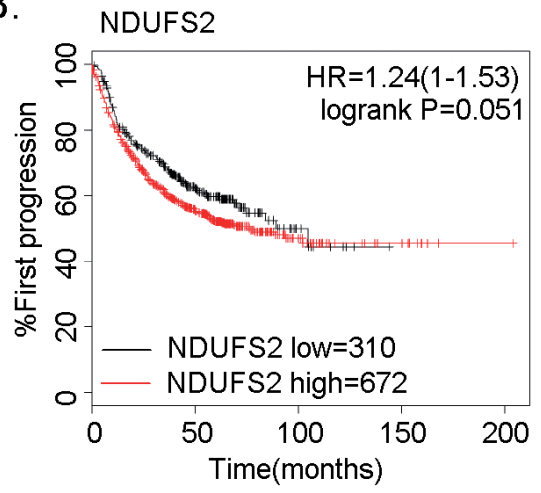

E.

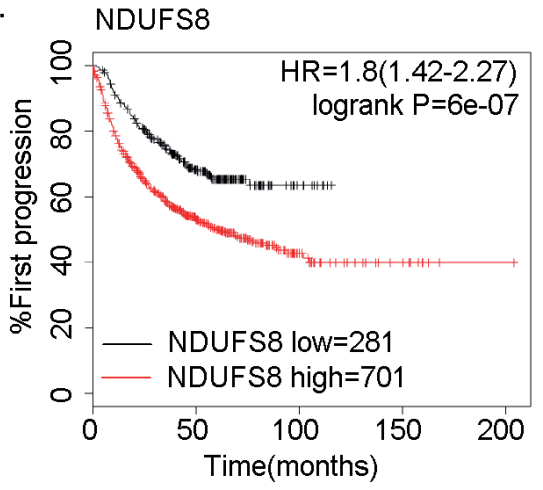

$\mathrm{H}$.

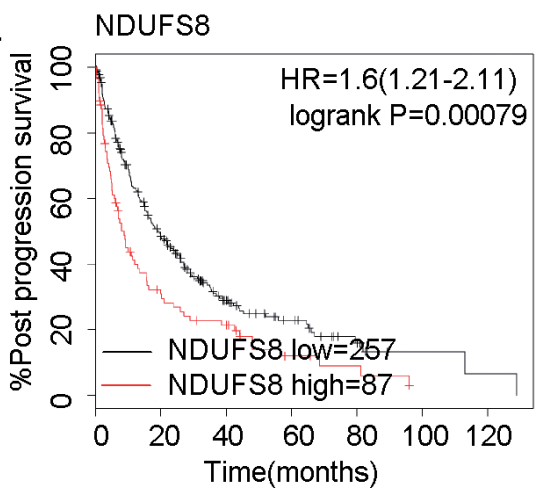

C.

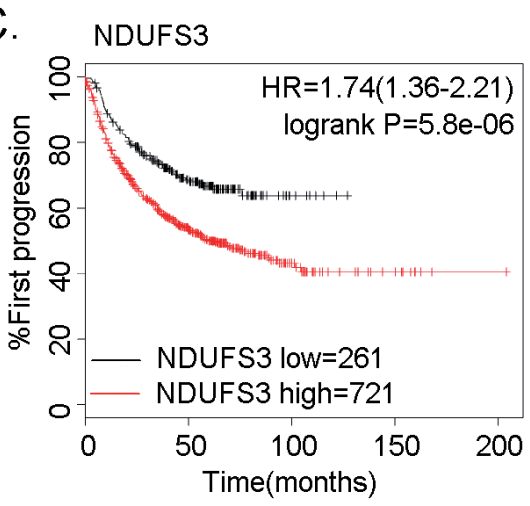

F.

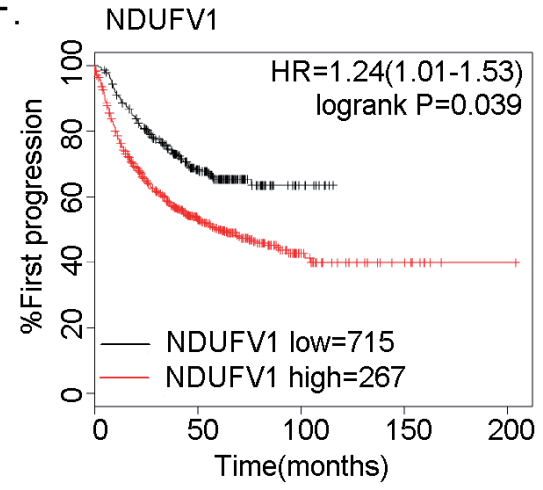

I.

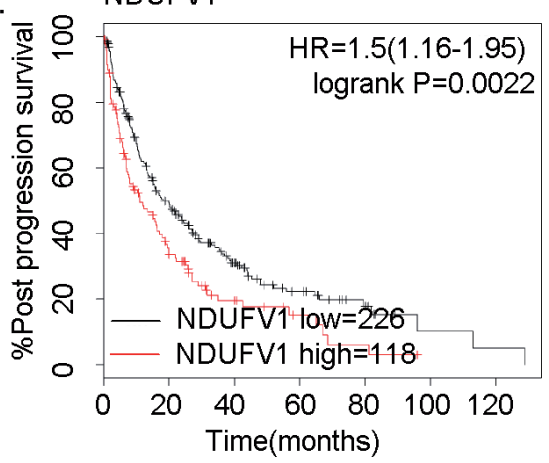

S-Figure 3. The prognostic value of the "core genes" expression in lung cancer patients

Notes: The desired Affymetrix ID of each gene in each tumor is valid, summarized in S-Table5. (a-i): Survival curves are plotted for all lung cancer patients of FP $(n=982)$, and PPS $(n=344)$. Data was analyzed using KM plotter (www.kmplot.com).

Abbreviation: HR: hazard ratio; FP: first progression; PPS: post progression survival. 
A.

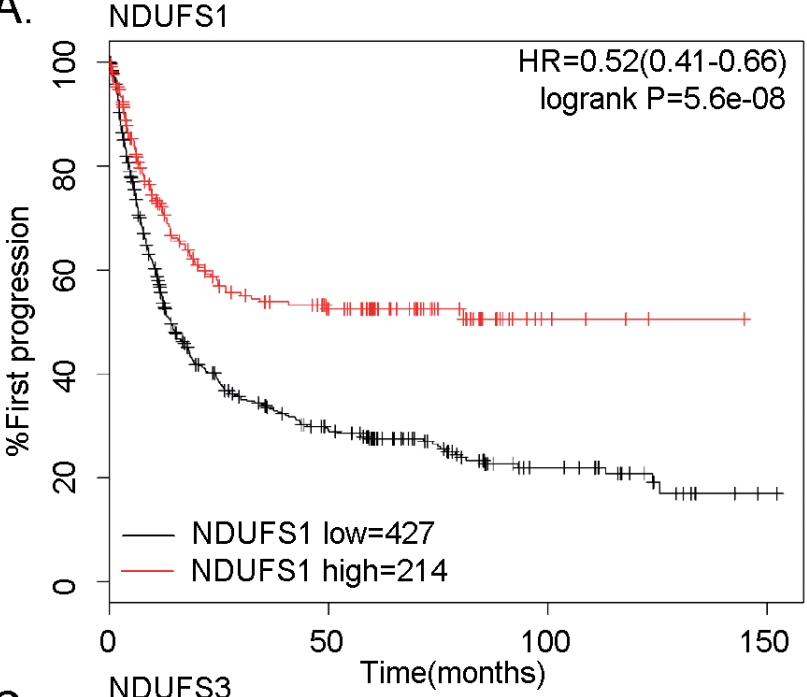

c.

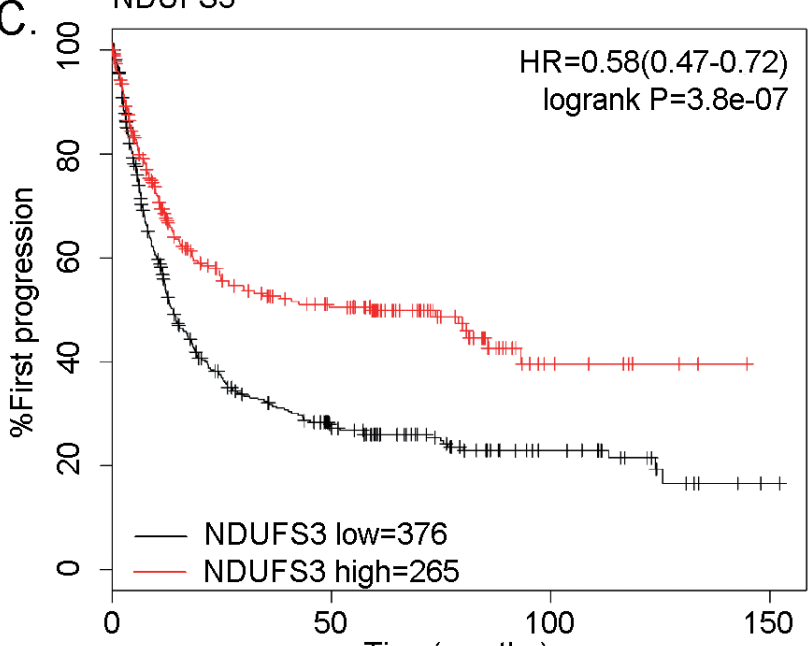

E.

Time(months)

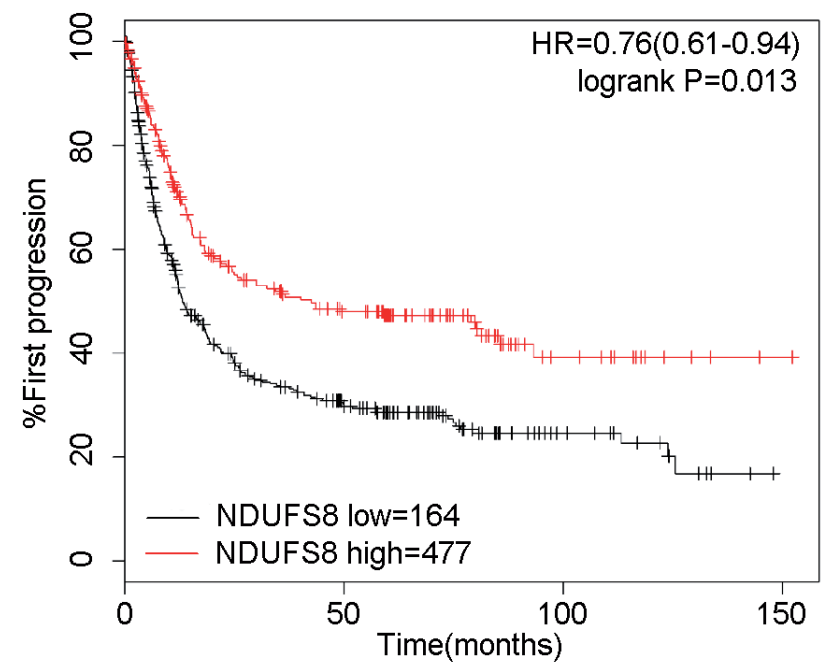

B.

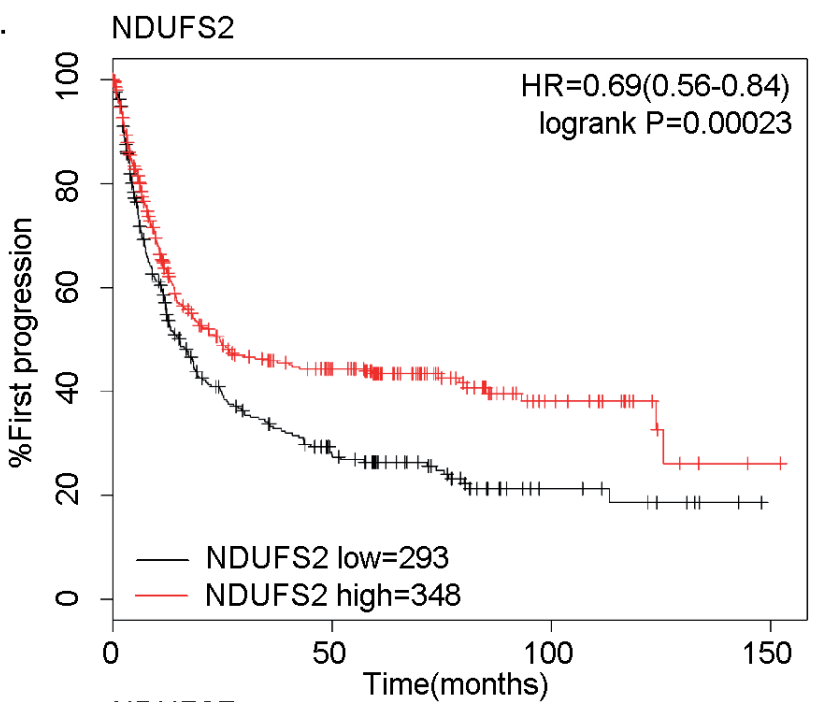

D.

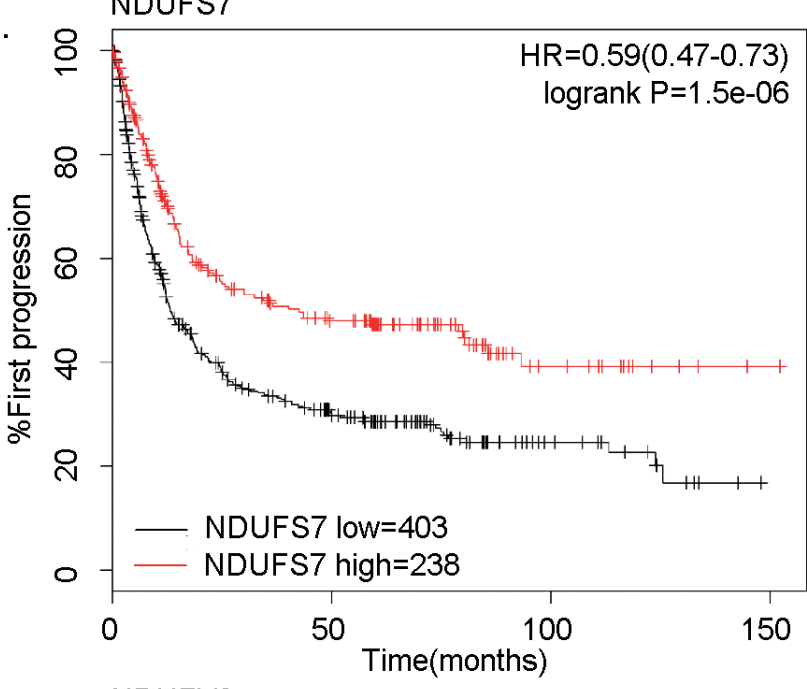

F.

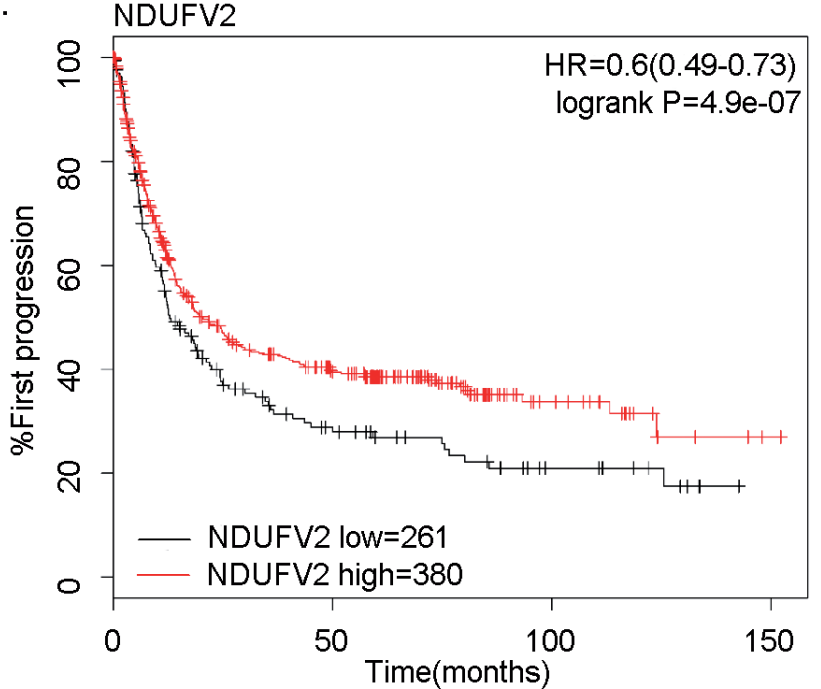

S-Figure 4. The prognostic value of the "core genes" expression in gastric cancer patients

Notes: The desired Affymetrix ID of each gene in each tumor is valid, summarized in S-Table5. (a-i): Survival curves are plotted for all gastric cancer patients of FP ( $n=982)$. Data was analyzed using Kaplan Meier plotter (www.kmplot.com).

Abbreviation: HR: hazard ratio; FP: first progression. 
- Cases with alterations in query genes

- Cases without alterations in query genes

A. All subunits for breast invasive carcinoma (TCGA, Nature 2012)

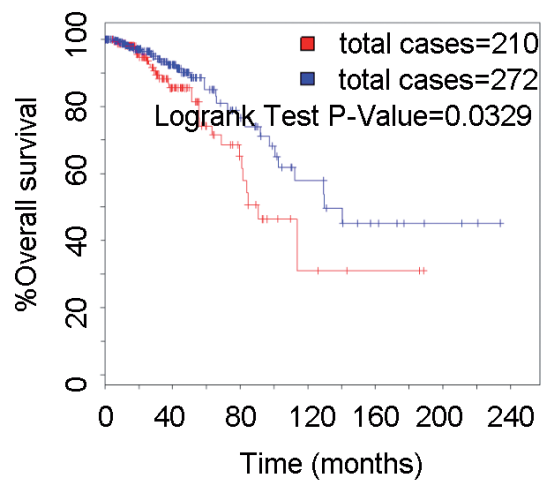

B. All subunits for ovarian serous

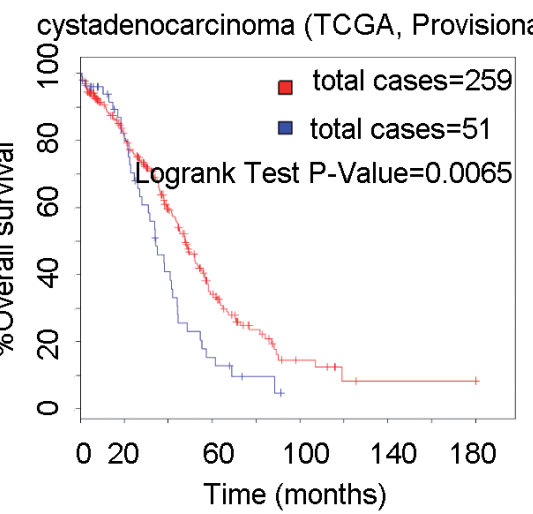

C. All subunits for esophageal squamous cell carcinoma (ICGC, Nature 2014)

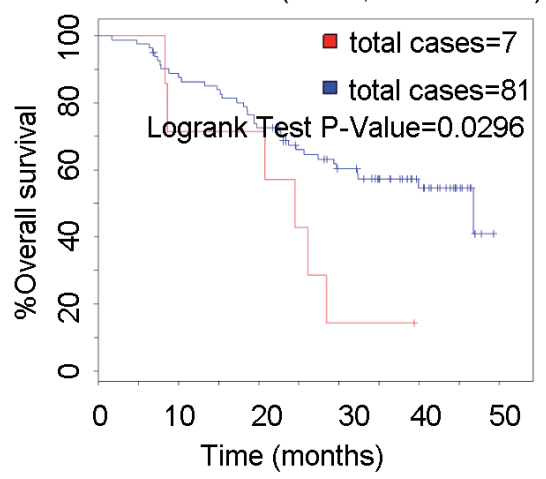

D. All subunits for acute myeloid leukemia (TCGA, Provisional)

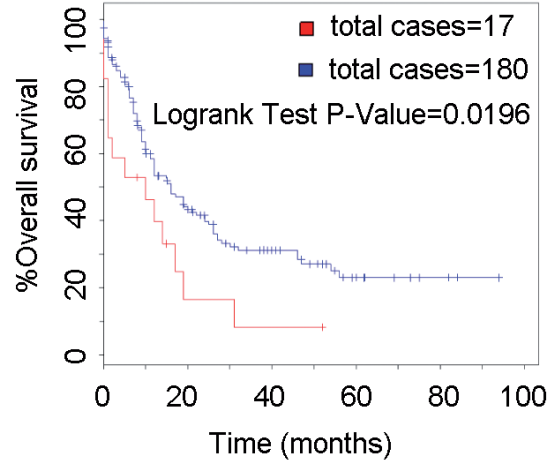

E. All subunits for bladder urothelial carcinoma (TCGA, Provisional)

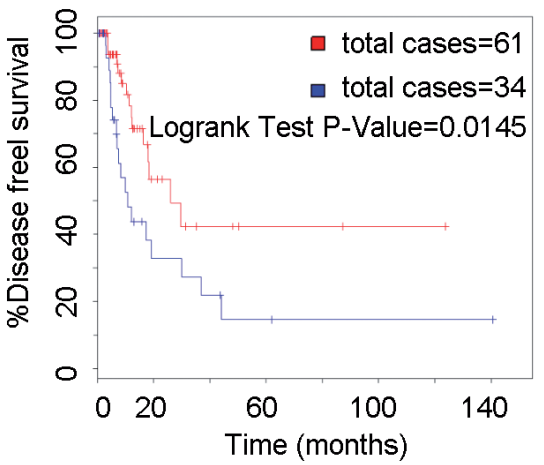

$F$.

Core subunits for prostate adenocarcinoma (TCGA, Provisional)

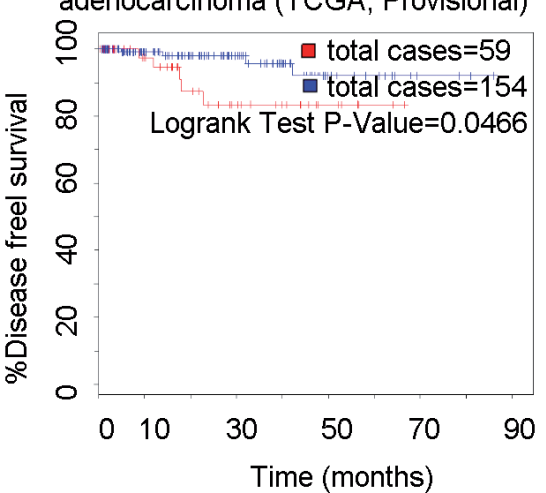

G. Core subunits for bladder urothelial carcinoma (TCGA, Nature 2014)

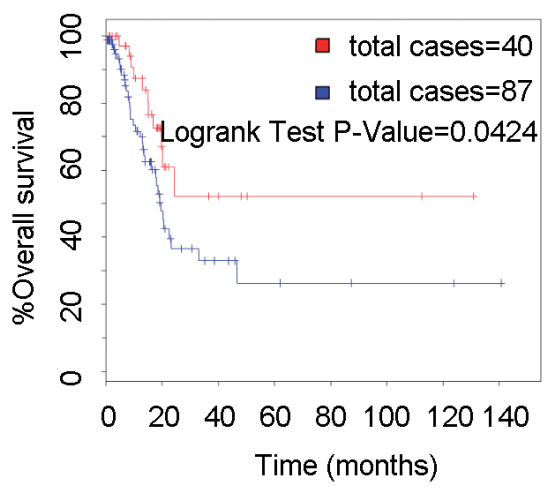

H. Core subunits for glioblastoma multiforme (TCGA, Provisional)

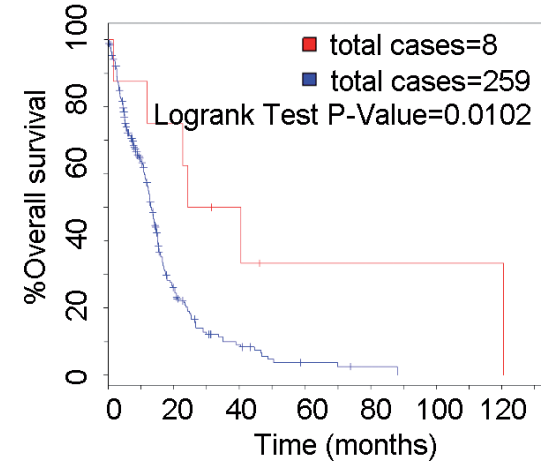

I. Core subunits for small cell

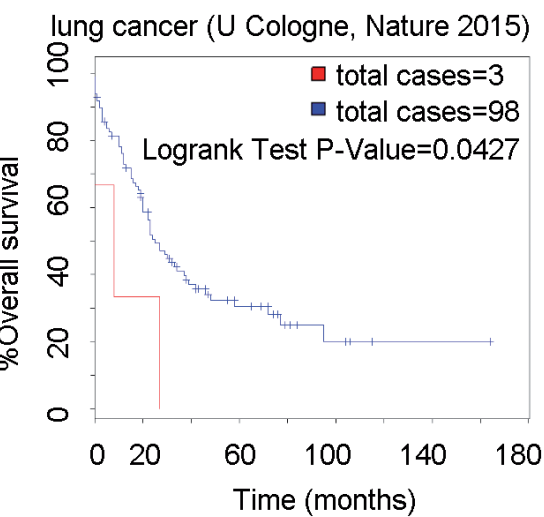

S-Figure 5. The significant prognostic value of the cumulative alteration of "all genes" and "core genes" in limited cancer patients Notes: Data was analyzed using cBio Cancer Genomics Portal (http://cbioportal.org).

Abbreviation: TCGA: the cancer genome atlas; ICGC: international cancer genome consortium. 
S-Table 1. The characteristic of the 14 core subunits of CI

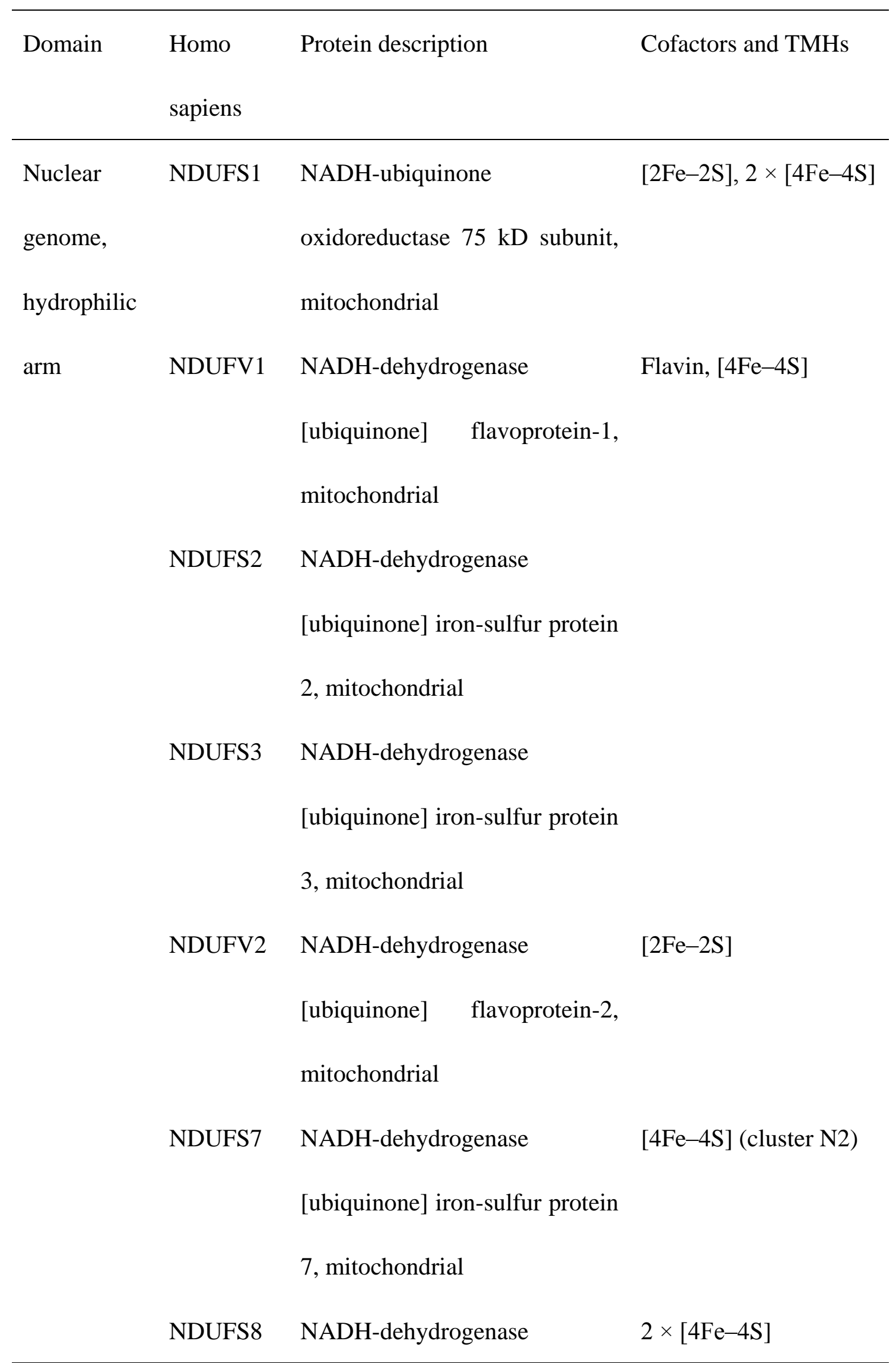


[ubiquinone] iron-sulfur protein

8, mitochondrial

\begin{tabular}{|c|c|c|c|}
\hline Mitochondri & ND1 & NADH-ubiquinone & $8 \mathrm{TMHs}^{\mathrm{a}}$ \\
\hline -al genome, & & oxidoreductase chain 1 & \\
\hline hydrophobi- & ND2 & NADH-ubiquinone & $14 \mathrm{TMHs}^{\mathrm{b}}$ \\
\hline \multirow[t]{10}{*}{$\mathrm{c}$ arm } & & oxidoreductase chain 2 & \\
\hline & ND3 & NADH-ubiquinone & 3 TMHs \\
\hline & & oxidoreductase chain 3 & \\
\hline & ND4 & NADH-ubiquinone & 14 TMHs \\
\hline & & oxidoreductase chain 4 & \\
\hline & ND4L & NADH-ubiquinone & 3 TMHs \\
\hline & & oxidoreductase chain $4 \mathrm{~L}$ & \\
\hline & ND5 & NADH-ubiquinone & $16 \mathrm{TMHs}$ \\
\hline & & oxidoreductase chain 5 & \\
\hline & ND6 & NADH-ubiquinone & $5 \mathrm{TMHs}$ \\
\hline
\end{tabular}

${ }^{\mathrm{a}}$ An extra C-terminal TMH is present in T. thermophilus.

${ }^{\mathrm{b}}$ Three TMHs are absent from the mammalian enzyme. 
S-Table 2. The six sub-complexes of total 49 mitochondrial complex I subunits.

Sub-complex Subunits

Alpha sub complex NDUFA1, NDUFA2, NDUFA3, NDUFA4, NDUFA5,

NDUFA6, NDUFA7, NDUFA8, NDUFA9, NDUFA10,

NDUFA11, NDUFA12, NDUFA13; NDUFAF1, NDUFAF2,

NDUFAF3, NDUFAF4; NDUFAB1

Beta sub complex NDUFB1 NDUFB2 NDUFB3 NDUFB4 NDUFB5 NDUFB6

NDUFB7 NDUFB8 NDUFB9 NDUFB10 NDUFB11

Sub complex

NDUFC1, NDUFC2

unknown

Flavoprotein $1 \quad$ NDUFV1, NDUFV2, NDUFV3

Fe-S protein NDUFS1, NDUFS2, NDUFS3, NDUFS4, NDUFS5, NDUFS6,

NDUFS7, NDUFS8

Mitochondrial

MT-ND1 MT-ND2 MT-ND3 MT-ND4 MT-ND4L MT-ND5

encoded NADH MT-ND6

dehydrogenase

subunit

S-Table3.1-3.12 were indicated in the $S$-Table3 file. 
S-Table3.1 Correlation of "core genes" with intrinsic subtypes of breast cancer patients.

\begin{tabular}{lllll}
\hline Gene & Intrinsic Subtypes & Case Number & HR $(95 \%$ CI $)$ & $P$ value \\
\hline NDUFS1 & Basal & 668 & $0.63(0.28-1.42)$ & 0.26 \\
& Luminal A & 2069 & $0.66(0.36-1.2)$ & 0.17 \\
& Luminal B & 1166 & $2.15(0.82-5.68)$ & 0.11 \\
& HER2+ & 239 & $1.75(0.69-4.44)$ & 0.24 \\
NDUFS2 & Basal & 668 & $0.58(0.33-1.01)$ & 0.051 \\
& Luminal A & 2069 & $0.85(0.58-1.25)$ & 0.41 \\
& Luminal B & 1166 & $1.52(0.99-2.32)$ & 0.053 \\
& HER2+ & 239 & $0.32(0.1-1.07)$ & 0.05 \\
NDUFS3 & Basal & 668 & $0.33(0.15-0.74)$ & 0.005 \\
& Luminal A & 2069 & $1.26(0.86-1.86)$ & 0.24 \\
& Luminal B & 1166 & $1.6(1.05-2.45)$ & 0.029 \\
& HER2+ & 239 & $1.41(0.65-3.08)$ & 0.39 \\
NDUFS7 & Basal & 668 & $1.93(1.11-3.36)$ & 0.018 \\
& Luminal A & 2069 & $1.35(0.87-2.1)$ & 0.18 \\
& Luminal B & 1166 & $1.59(1.03-2.46)$ & 0.033 \\
& HER2+ & 239 & $1.85(0.86-3.95)$ & 0.11 \\
NDUFS8 & Basal & 668 & $0.75(0.43-1.31)$ & 0.31 \\
& Luminal A & 2069 & $1.43(0.95-2.15)$ & 0.087 \\
& Luminal B & 1166 & $1.69(1.07-2.67)$ & 0.022 \\
& HER2+ & 239 & $0.68(0.32-1.47)$ & 0.33 \\
NDUFV1 & Basal & 668 & $0.76(0.44-1.32)$ & 0.33 \\
& Luminal A & 2069 & $1.5(1.01-2.21)$ & 0.042 \\
& Luminal B & 1166 & $1.52(0.99-2.34)$ & 0.056 \\
& HER2+ & 239 & $4.51(1.07-19.05$ & 0.025 \\
& Basal & 668 & $1.43(0.75-2.73)$ & 0.28 \\
& Luminal A & 2069 & $0.51(0.35-0.75)$ & 0.00053 \\
& Luminal B & 1166 & $1.51(0.97-2.35)$ & 0.068 \\
& HER2+ & 239 & $0.55(0.25-1.21)$ & 0.13 \\
\hline
\end{tabular}


S-Table3.2 Correlation of "core genes" with pathology grades of breast cancer patients.

\begin{tabular}{|c|c|c|c|c|}
\hline Gene & Grade & Case Number & $\mathrm{HR}(95 \% \mathrm{CI})$ & $P$ value \\
\hline \multirow[t]{3}{*}{ NDUFS1 } & I & 318 & $0(0-$ Inf $)$ & 0.28 \\
\hline & II & 778 & $3.54(0.34-37.02)$ & 0.27 \\
\hline & III & 811 & $0.52(0.22-1.24)$ & 0.13 \\
\hline \multirow[t]{3}{*}{ NDUFS2 } & I & 318 & $1.69(0.64-4.45)$ & 0.28 \\
\hline & II & 778 & $1.64(1.03-2.61)$ & 0.034 \\
\hline & III & 811 & $0.85(0.57-1.27)$ & 0.44 \\
\hline \multirow[t]{3}{*}{ NDUFS3 } & I & 318 & $3.48(1.26-9.62)$ & 0.01 \\
\hline & II & 778 & $1.53(0.9-2.58)$ & 0.11 \\
\hline & III & 811 & $0.69(0.45-1.08)$ & 0.1 \\
\hline \multirow[t]{3}{*}{ NDUFS7 } & I & 318 & $6.35(0.84-48.15)$ & 0.041 \\
\hline & II & 778 & $2.18(1.28-3.73)$ & 0.0033 \\
\hline & III & 811 & $1.6(1.06-2.42)$ & 0.025 \\
\hline \multirow[t]{3}{*}{ NDUFS8 } & I & 318 & $4.55(1.75-11.81)$ & 0.00066 \\
\hline & II & 778 & $1.8(1.05-3.11)$ & 0.031 \\
\hline & III & 811 & $1.4(0.87-2.26)$ & 0.16 \\
\hline \multirow[t]{3}{*}{ NDUFV1 } & $\mathrm{I}$ & 318 & $4.55(1.68-12.34)$ & 0.0011 \\
\hline & II & 778 & $2.09(1.16-3.76)$ & 0.012 \\
\hline & III & 811 & $1.18(0.78-1.79)$ & 0.43 \\
\hline \multirow[t]{3}{*}{ NDUFV2 } & I & 318 & $1.71(0.66-4.42)$ & 0.26 \\
\hline & II & 778 & $1.42(0.88-2.27)$ & 0.15 \\
\hline & III & 811 & $0.67(0.45-1.01)$ & 0.055 \\
\hline
\end{tabular}


S-Table3.3 Correlation of "core genes" with pathology grades of ovarian cancer patients.

\begin{tabular}{|c|c|c|c|c|}
\hline Gene & Grade & Case Number & $\mathrm{HR}(95 \% \mathrm{CI})$ & $P$ value \\
\hline \multirow[t]{3}{*}{ NDUFS1 } & I & 56 & $0.43(0.12-1.5)$ & 0.17 \\
\hline & II & 315 & $1.52(0.84-2.77)$ & 0.16 \\
\hline & III & 974 & $0.89(0.66-1.19)$ & 0.41 \\
\hline \multirow[t]{3}{*}{ NDUFS2 } & I & 56 & $2.7(1.03-7.04)$ & 0.035 \\
\hline & II & 315 & $0.77(0.56-1.04)$ & 0.09 \\
\hline & III & 974 & $1.39(1.15-1.66)$ & 0.00045 \\
\hline \multirow[t]{3}{*}{ NDUFS3 } & I & 56 & $0.46(0.18-1.18)$ & 0.099 \\
\hline & II & 315 & $0.65(0.46-0.92)$ & 0.015 \\
\hline & III & 974 & $0.82(0.69-0.97)$ & 0.022 \\
\hline \multirow[t]{3}{*}{ NDUFS7 } & I & 56 & $0.38(0.14-1.02)$ & 0.045 \\
\hline & II & 315 & $0.68(0.49-0.96)$ & 0.026 \\
\hline & III & 974 & $0.88(0.74-1.05)$ & 0.14 \\
\hline \multirow[t]{3}{*}{ NDUFS8 } & I & 56 & $2.13(0.84-5.41)$ & 0.1 \\
\hline & II & 315 & $0.64(0.47-0.88)$ & 0.0051 \\
\hline & III & 974 & $0.88(0.72-1.06)$ & 0.18 \\
\hline \multirow[t]{3}{*}{ NDUFV1 } & I & 56 & $3.62(1.33-9.83)$ & 0.007 \\
\hline & II & 315 & $0.69(0.51-0.95)$ & 0.023 \\
\hline & III & 974 & $0.86(0.71-1.02)$ & 0.089 \\
\hline \multirow[t]{3}{*}{ NDUFV2 } & I & 56 & $0.7(0.26-1.86)$ & 0.47 \\
\hline & II & 315 & $0.68(0.5-0.93)$ & 0.016 \\
\hline & III & 974 & $0.85(0.71-1.03)$ & 0.093 \\
\hline
\end{tabular}


S-Table3.4 Correlation of "core genes" with histologic types of ovarian cancer patients.

\begin{tabular}{lllll}
\hline Gene & Histologic Type & Case Number & HR $(95 \% \mathrm{CI})$ & $P$ value \\
\hline NDUFS1 & Endometrioid & 36 & $0(0-$ Inf $)$ & 0.085 \\
& Serous & 1144 & $1.12(0.84-1.5)$ & 0.43 \\
NDUFS2 & Endometrioid & 36 & $0(0-$ Inf $)$ & 0.039 \\
& Serous & 1144 & $1.37(1.15-1.64)$ & 0.00042 \\
NDUFS3 & Endometrioid & 36 & $0(0-$ Inf $)$ & 0.11 \\
& Serous & 1144 & $0.84(0.71-0.99)$ & 0.038 \\
NDUFS7 & Endometrioid & 36 & $2.7(0.45-16.15)$ & 0.26 \\
& Serous & 1144 & $0.88(0.75-1.03)$ & 0.11 \\
NDUFS8 & Endometrioid & 36 & $0.36(0.06-2.18)$ & 0.25 \\
& Serous & 1144 & $0.84(0.71-1)$ & 0.049 \\
NDUFV1 & Endometrioid & 36 & $0(0-$ Inf $)$ & 0.15 \\
& Serous & 1144 & $0.86(0.72-1.01)$ & 0.071 \\
NDUFV2 & Endometrioid & 36 & $2.62(0.44-15.68)$ & 0.27 \\
& Serous & 1144 & $0.84(0.71-0.99)$ & 0.037 \\
\hline
\end{tabular}

S-Table3.5 Correlation of "core genes" with histologic types of lung cancer patients.

\begin{tabular}{lllll}
\hline Gene & Histologic Type & Case Number & HR $(95 \% \mathrm{CI})$ & $P$ value \\
\hline NDUFS1 & adenocarcinoma & 866 & $0.39(0.3-0.51)$ & $7.7 \mathrm{e}-13$ \\
& squamous cell carcinoma & 675 & $0.7(0.5-0.98)$ & 0.035 \\
NDUFS2 & adenocarcinoma & 866 & $1.35(1.05-1.74)$ & 0.018 \\
& squamous cell carcinoma & 675 & $0.91(0.71-1.17)$ & 0.48 \\
NDUFS3 & adenocarcinoma & 866 & $2.98(2.09-4.26)$ & $2.3 \mathrm{e}-10$ \\
& squamous cell carcinoma & 675 & $1.29(0.99-1.67)$ & 0.056 \\
NDUFS7 & adenocarcinoma & 866 & $1.52(1.2-1.92)$ & 0.00039 \\
& squamous cell carcinoma & 675 & $0.78(0.6-1.03)$ & 0.074 \\
NDUFS8 & adenocarcinoma & 866 & $3.29(2.31-4.67)$ & $1.9 \mathrm{e}-12$ \\
& squamous cell carcinoma & 675 & $1.49(1.1-2.02)$ & 0.0092 \\
NDUFV1 & adenocarcinoma & 866 & $1.44(1.14-1.82)$ & 0.0018 \\
& squamous cell carcinoma & 675 & $1.31(1.02-1.69)$ & 0.034 \\
NDUFV2 & adenocarcinoma & 866 & $2.77(2.01-3.83)$ & $1.1 \mathrm{e}-10$ \\
& squamous cell carcinoma & 675 & $1.23(0.97-1.57)$ & 0.087 \\
\hline
\end{tabular}


S-Table3.6 Correlation of "core genes" with clinical stage of lung cancer patients.

\begin{tabular}{lclll}
\hline Gene & Clinical Stage & Case Number & HR $(95 \%$ CI $)$ & $P$ value \\
\hline NDUFS1 & I & 652 & $0.34(0.25-0.48)$ & $2.8 \mathrm{e}-11$ \\
& II & 320 & $0.58(0.36-0.94)$ & 0.025 \\
NDUFS2 & III & 70 & $0.66(0.31-1.39)$ & 0.27 \\
& I & 652 & $1.78(1.34-2.36)$ & $4.9 \mathrm{e}-05$ \\
NDUFS3 & II & 320 & $0.7(0.48-1.02)$ & 0.061 \\
& I & 70 & $0.72(0.39-1.32)$ & 0.28 \\
& II & 652 & $2.91(2.07-4.09)$ & $1.4 \mathrm{e}-10$ \\
NDUFS7 & III & 320 & $1.53(1.06-2.2)$ & 0.023 \\
& I & 70 & $0.68(0.37-1.26)$ & 0.22 \\
& II & 652 & $3.06(2.08-4.5)$ & $2.6 \mathrm{e}-09$ \\
NDUFS8 & III & 320 & $1.64(1.05-2.56)$ & 0.027 \\
& I & 70 & $1.52(0.86-2.7)$ & 0.15 \\
& II & 652 & $3.68(2.48-5.45)$ & $3.4 \mathrm{e}-12$ \\
NDUFV1 & III & 320 & $2.28(1.45-3.58)$ & 0.00024 \\
& I & 70 & $1.64(0.94-2.84)$ & 0.076 \\
& II & 652 & $1.97(1.46-2.66)$ & $6.5 \mathrm{e}-06$ \\
& III & 320 & $1.45(1-2.1)$ & 0.046 \\
NDUFV2 & I & 70 & $0.71(0.4-1.28)$ & 0.25 \\
& II & 652 & $2.98(2.21-4)$ & $4.2 \mathrm{e}-14$ \\
& III & 320 & $1.77(1.2-2.6)$ & 0.0032 \\
& & 70 & $1.39(0.78-2.46)$ & 0.26 \\
\hline
\end{tabular}

S-Table3.7 Correlation of "core genes" with gender of lung cancer patients.

\begin{tabular}{lllll}
\hline Gene & Gender & Case Number & HR $(95 \%$ CI $)$ & $P$ value \\
\hline NDUFS1 & Female & 818 & $0.34(0.24-0.48)$ & $1.4 \mathrm{e}-10$ \\
& Male & 1387 & $0.51(0.41-0.65)$ & $1 \mathrm{e}-08$ \\
NDUFS2 & Female & 818 & $1.43(1.12-1.84)$ & 0.0041 \\
& Male & 1387 & $1.08(0.92-1.26)$ & 0.36 \\
NDUFS3 & Female & 818 & $1.56(1.16-2.09)$ & 0.0031 \\
& Male & 1387 & $1.54(1.27-1.88)$ & $1.2 \mathrm{e}-05$ \\
NDUFS7 & Female & 818 & $1.56(1.19-2.04)$ & 0.0012 \\
& Male & 1387 & $1.26(1.04-1.52)$ & 0.016 \\
NDUFS8 & Female & 818 & $1.97(1.43-2.72)$ & $2.7 \mathrm{e}-05$ \\
& Male & 1387 & $1.67(1.35-2.05)$ & $1.3 \mathrm{e}-06$ \\
NDUFV1 & Female & 818 & $1.41(1.1-1.8)$ & 0.0065 \\
& Male & 1387 & $1.22(1.04-1.43)$ & 0.014 \\
NDUFV2 & Female & 818 & $2.18(1.58-3)$ & $1 \mathrm{e}-06$ \\
& Male & 1387 & $1.64(1.35-2)$ & $4.8 \mathrm{e}-07$ \\
\hline
\end{tabular}


S-Table3.8 Correlation of "core genes" with smoking history of lung cancer patients.

\begin{tabular}{lllll}
\hline Gene & Smoking History & Case Number & HR $(95 \%$ CI $)$ & $P$ value \\
\hline NDUFS1 & Smokers & 970 & $0.62(0.41-0.95)$ & 0.026 \\
& Non-smokers & 247 & $0.34(0.1-1.16)$ & 0.071 \\
NDUFS2 & Smokers & 970 & $1.47(1.19-1.81)$ & 0.00032 \\
& Non-smokers & 247 & $1.65(0.84-3.23)$ & 0.14 \\
NDUFS3 & Smokers & 970 & $1.58(1.2-2.07)$ & 0.0011 \\
& Non-smokers & 247 & $3.26(1.84-5.78)$ & $1.9 \mathrm{e}-05$ \\
NDUFS7 & Smokers & 970 & $0.83(0.67-1.04)$ & 0.099 \\
& Non-smokers & 247 & $3.89(2.15-7.06)$ & $1.5 \mathrm{e}-06$ \\
NDUFS8 & Smokers & 970 & $1.61(1.25-2.08)$ & 0.00023 \\
& Non-smokers & 247 & $4.37(2.28-8.38)$ & $1.2 \mathrm{e}-06$ \\
NDUFV1 & Smokers & 970 & $1.27(1.03-1.57)$ & 0.027 \\
& Non-smokers & 247 & $2.33(1.31-4.14)$ & 0.0029 \\
NDUFV2 & Smokers & 970 & $1.67(1.33-2.09)$ & $7.5 \mathrm{e}-06$ \\
& Non-smokers & 247 & $3.74(2.06-6.79)$ & $3.2 \mathrm{e}-06$ \\
\hline
\end{tabular}


S-Table3.9 Correlation of "core genes" with clinical stage of gastric cancer patients.

\begin{tabular}{|c|c|c|c|c|}
\hline Gene & Clinical Stage & Case Number & $\mathrm{HR}(95 \% \mathrm{CI})$ & $P$ value \\
\hline \multirow[t]{4}{*}{ NDUFS1 } & $\mathrm{I}$ & 69 & $0.16(0.05-0.57)$ & 0.0014 \\
\hline & II & 145 & $0.69(0.36-1.36)$ & 0.28 \\
\hline & III & 319 & $0.57(0.42-0.76)$ & $9.3 \mathrm{e}-05$ \\
\hline & IV & 152 & $0.63(0.43-0.93)$ & 0.019 \\
\hline \multirow[t]{4}{*}{ NDUFS2 } & I & 69 & $0.46(0.17-1.26)$ & 0.12 \\
\hline & II & 145 & $0.62(0.34-1.13)$ & 0.11 \\
\hline & III & 319 & $0.57(0.42-0.77)$ & 0.00024 \\
\hline & IV & 152 & $0.67(0.46-1)$ & 0.047 \\
\hline \multirow[t]{4}{*}{ NDUFS3 } & I & 69 & $1.68(0.54-5.27)$ & 0.37 \\
\hline & II & 145 & $0.44(0.24-0.8)$ & 0.0062 \\
\hline & III & 319 & $0.72(0.52-0.99)$ & 0.043 \\
\hline & IV & 152 & $0.51(0.34-0.77)$ & 0.001 \\
\hline \multirow[t]{4}{*}{ NDUFS7 } & I & 69 & $0.36(0.12-1.04)$ & 0.048 \\
\hline & II & 145 & $0.41(0.2-0.84)$ & 0.011 \\
\hline & III & 319 & $0.62(0.46-0.83)$ & 0.0013 \\
\hline & IV & 152 & $0.63(0.41-0.98)$ & 0.038 \\
\hline \multirow[t]{4}{*}{ NDUFS8 } & I & 69 & $0.45(0.17-1.24)$ & 0.11 \\
\hline & II & 145 & $1.7(0.94-3.08)$ & 0.075 \\
\hline & III & 319 & $0.67(0.5-0.91)$ & 0.011 \\
\hline & IV & 152 & $0.65(0.43-0.98)$ & 0.036 \\
\hline \multirow[t]{4}{*}{ NDUFV1 } & I & 69 & $2.6(0.92-7.37)$ & 0.064 \\
\hline & II & 145 & $1.9(0.88-4.11)$ & 0.097 \\
\hline & III & 319 & $1.83(1.37-2.44)$ & $2.8 \mathrm{e}-05$ \\
\hline & IV & 152 & $0.71(0.45-1.12)$ & 0.14 \\
\hline \multirow[t]{4}{*}{ NDUFV2 } & I & 69 & $0.35(0.12-1.03)$ & 0.047 \\
\hline & II & 145 & $0.31(0.14-0.69)$ & 0.0025 \\
\hline & III & 319 & $0.54(0.39-0.74)$ & 0.00011 \\
\hline & IV & 152 & $0.59(0.4-0.87$ & 0.0068 \\
\hline
\end{tabular}


S-Table3.10 Correlation of "core genes" with differentiated degree of gastric cancer patients.

\begin{tabular}{lllll}
\hline Gene & Differentiation & Case Number & HR $(95 \%$ CI $)$ & $P$ value \\
\hline NDUFS1 & poorly & 166 & $1.4(0.93-2.11)$ & 0.1 \\
& moderately & 67 & $0.73(0.37-1.43)$ & 0.35 \\
& well & 32 & $0.23(0.08-0.7)$ & 0.0047 \\
NDUFS2 & poorly & 166 & $1.59(0.97-2.6)$ & 0.065 \\
& moderately & 67 & $0.73(0.36-1.48)$ & 0.38 \\
& well & 32 & $0.39(0.16-0.98)$ & 0.037 \\
NDUFS3 & poorly & 166 & $1.46(0.98-2.18)$ & 0.064 \\
& moderately & 67 & $0.46(0.2-1.05)$ & 0.059 \\
& well & 32 & $0.47(0.19-1.21)$ & 0.11 \\
NDUFS7 & poorly & 166 & $1.3(0.81-2.07)$ & 0.27 \\
& moderately & 67 & $0.46(0.24-0.9)$ & 0.02 \\
& well & 32 & $0.61(0.22-1.69)$ & 0.34 \\
NDUFS8 & poorly & 166 & $0.66(0.4-1.1)$ & 0.11 \\
& moderately & 67 & $0.41(0.73-2.72)$ & 0.3 \\
& well & 32 & $0.25(0.1-0.65)$ & 0.0023 \\
NDUFV1 & poorly & 166 & $1.39(0.93-2.08)$ & 0.11 \\
& moderately & 67 & $0.5(0.26-0.96)$ & 0.034 \\
& well & 32 & $2.24(0.75-6.66)$ & 0.14 \\
NDUFV2 & poorly & 166 & $0.64(0.39-1.08)$ & 0.091 \\
& moderately & 67 & $0.65(0.33-1.31)$ & 0.23 \\
& well & 32 & $1.93(0.8-4.66)$ & 0.14 \\
\hline
\end{tabular}

S-Table3.11 Correlation of "core genes" with gender of gastric cancer patients.

\begin{tabular}{lllll}
\hline Gene & Gender & Case Number & HR $(95 \% \mathrm{CI})$ & $P$ value \\
\hline NDUFS1 & Female & 244 & $0.35(0.22-0.58)$ & $1.3 \mathrm{e}-05$ \\
& Male & 567 & $0.49(0.38-0.64)$ & $3.2 \mathrm{e}-08$ \\
NDUFS2 & Female & 244 & $0.65(0.46-0.93)$ & 0.017 \\
& Male & 567 & $0.65(0.52-0.8)$ & $6.9 \mathrm{e}-05$ \\
NDUFS3 & Female & 244 & $0.64(0.45-0.92)$ & 0.016 \\
& Male & 567 & $0.64(0.52-0.8)$ & $9.7 \mathrm{e}-05$ \\
NDUFS7 & Female & 244 & $0.44(0.28-0.67)$ & 0.00013 \\
& Male & 567 & $0.65(0.52-0.8)$ & $5.7 \mathrm{e}-05$ \\
NDUFS8 & Female & 244 & $0.58(0.4-0.84)$ & 0.0036 \\
& Male & 567 & $0.71(0.56-0.9)$ & 0.0037 \\
NDUFV1 & Female & 244 & $1.53(1.07-2.18)$ & 0.018 \\
& Male & 567 & $1.63(1.31-2.03)$ & $7.6 \mathrm{e}-06$ \\
NDUFV2 & Female & 244 & $0.5(0.34-0.74)$ & 0.00038 \\
& Male & 567 & $0.54(0.44-0.67)$ & $2.4 \mathrm{e}-08$ \\
\hline
\end{tabular}


S-Table3.12 Correlation of "core genes" with Lauren Classification of gastric cancer patients.

\begin{tabular}{lllll}
\hline Gene & Lauren Classification & Case Number & HR $(95 \% \mathrm{CI})$ & $P$ value \\
\hline NDUFS1 & Intestinal & 336 & $0.41(0.28-0.58)$ & $2.5 \mathrm{e}-07$ \\
& Diffuse & 248 & $0.59(0.42-0.84)$ & 0.0026 \\
& Mixed & 33 & $0.5(0.16-1.56)$ & 0.22 \\
NDUFS2 & Intestinal & 336 & $0.56(0.41-0.77)$ & 0.00026 \\
& Diffuse & 248 & $0.56(0.4-0.79)$ & 0.00075 \\
& Mixed & 33 & $0.52(0.17-1.54)$ & 0.23 \\
NDUFS3 & Intestinal & 336 & $0.52(0.38-0.71)$ & $4.2 \mathrm{e}-05$ \\
& Diffuse & 248 & $0.61(0.4-0.93)$ & 0.021 \\
& Mixed & 33 & $0.39(0.14-1.12)$ & 0.071 \\
NDUFS7 & Intestinal & 336 & $0.59(0.42-0.83)$ & 0.0019 \\
& Diffuse & 248 & $0.61(0.43-0.87)$ & 0.0054 \\
& Mixed & 33 & $2.41(0.67-8.61)$ & 0.16 \\
NDUFS8 & Intestinal & 336 & $0.65(0.46-0.9)$ & 0.0089 \\
& Diffuse & 248 & $0.8(0.55-1.17)$ & 0.26 \\
& Mixed & 33 & $0.22(0.06-0.8)$ & 0.012 \\
NDUFV1 & Intestinal & 336 & $1.93(1.36-2.75)$ & $2 \mathrm{e}-04$ \\
& Diffuse & 248 & $1.31(0.93-1.84)$ & 0.12 \\
& Mixed & 33 & $2.03(0.73-5.62)$ & 0.17 \\
& Intestinal & 336 & $0.41(0.3-0.56)$ & $1.2 \mathrm{e}-08$ \\
& Diffuse & 248 & $0.59(0.42-0.83)$ & 0.0023 \\
& Mixed & 33 & $0.39(0.12-1.24)$ & 0.098 \\
\hline
\end{tabular}


S-Table 4. The summary of desired Affymetrix ID, Cases Number, Hazard Ratio (HR; and $95 \%$ confidence intervals) and Log Rank P of the individual all gene's OS curves for the four different tumors.

\begin{tabular}{|c|c|c|c|c|c|c|c|c|}
\hline Cancer & Subunits & Affymetrix ID & Survival & Cases & Cases & HR & $95 \% \mathrm{CI}$ & P-value \\
\hline & & & & -low & -high & & & \\
\hline Breast & NDUFA2 & 209223_at & OS & 341 & 776 & 1.33 & $1.02-1.47$ & 0.0368 \\
\hline \multirow[t]{16}{*}{ cancer } & NDUFA4 & 217773_s_at & OS & 820 & 297 & 1.54 & $1.19-1.99$ & 0.00083 \\
\hline & NDUFA6 & 202001_s_at & OS & 456 & 661 & 1.51 & $1.17-2.93$ & 0.0012 \\
\hline & NDUFA8 & 218160_at & OS & 354 & 763 & 1.33 & $1.02-1.74$ & 0.038 \\
\hline & NDUFA9 & 208969_at & OS & 547 & 570 & 1.3 & $1.03-1.65$ & 0.029 \\
\hline & NDUFAF2 & 228355_s_at & OS & 361 & 161 & 1.59 & $1.09-2.32$ & 0.014 \\
\hline & NDUFAF4 & 219006_at & OS & 632 & 485 & 1.6 & $1.26-2.04$ & 0.0001 \\
\hline & NDUFAB1 & 202077_at & OS & 685 & 432 & 1.41 & $1.11-1.78$ & 0.0049 \\
\hline & NDUFB2 & 218200_s_at & OS & 444 & 673 & 1.36 & $1.06-1.74$ & 0.015 \\
\hline & NDUFB3 & 203371_s_at & OS & 329 & 788 & 1.33 & $1.02-1.74$ & 0.036 \\
\hline & NDUFB4 & 218226_s_at & OS & 737 & 380 & 1.38 & $1.09-1.76$ & 0.0082 \\
\hline & NDUFB5 & 203621_at & OS & 825 & 292 & 1.33 & $1.02-1.74$ & 0.035 \\
\hline & NDUFB6 & 203613_s_at & OS & 461 & 656 & 1.49 & $1.16-1.91$ & 0.0016 \\
\hline & NDUFB7 & 211407_at & OS & 570 & 547 & 1.32 & $1.04-1.67$ & 0.023 \\
\hline & NDUFB9 & 222992_s_at & OS & 227 & 295 & 1.46 & $1.02-2.11$ & 0.04 \\
\hline & NDUFB11 & 218320_s_at & OS & 516 & 601 & 1.35 & $1.06-1.72$ & 0.015 \\
\hline & NDUFC2 & 218101_s_at & OS & 815 & 302 & 1.34 & $1.04-1.73$ & 0.024 \\
\hline
\end{tabular}




\begin{tabular}{|c|c|c|c|c|c|c|c|c|}
\hline & NDUFS6 & 203606_at & OS & 458 & 659 & 1.8 & $1.39-2.32$ & $6.2 E-06$ \\
\hline & NDUFV3 & 226209_at & OS & 171 & 351 & 1.59 & $1.06-2.39$ & 0.024 \\
\hline & NDUFB8 & 201227_s_at & OS & 278 & 839 & 0.71 & $0.55-0.91$ & 0.0073 \\
\hline & NDUFA1 & 202298_at & OS & 532 & 585 & 1.26 & $0.99-1.6$ & 0.06 \\
\hline & NDUFA3 & 218563_at & OS & 405 & 712 & 1.16 & $0.9-1.5$ & 0.25 \\
\hline & NDUFA11 & 228690_s_at & OS & 309 & 213 & 1.39 & $0.97-1.98$ & 0.069 \\
\hline & NDUFA12 & 223244_s_at & OS & 273 & 249 & 1.42 & $0.99-2.03$ & 0.052 \\
\hline & NDUFA13 & 220864_s_at & OS & 836 & 281 & 1.27 & $0.98-1.65$ & 0.068 \\
\hline & NDUFB10 & 223112_s_at & OS & 338 & 184 & 1.22 & $0.85-1.76$ & 0.28 \\
\hline & NDUFC1 & 203478_at & OS & 732 & 385 & 1.07 & $0.84-1.37$ & 0.58 \\
\hline & NDUFS5 & 201757_at & OS & 430 & 687 & 1.28 & $0.99-1.64$ & 0.057 \\
\hline & NDUFA5 & 215850_s_at & OS & 305 & 812 & 0.82 & $0.63-1.06$ & 0.12 \\
\hline & NDUFA7 & 1557532_at & OS & 151 & 371 & 0.7 & $0.48-1.01$ & 0.057 \\
\hline & NDUFA10 & 217860_at & OS & 580 & 537 & 0.82 & $0.64-1.04$ & 0.093 \\
\hline & NDUFAF1 & 204125_at & OS & 305 & 812 & 0.78 & $0.6-1$ & 0.053 \\
\hline & NDUFAF3 & 209177_at & OS & 305 & 812 & 0.83 & $0.64-1.07$ & 0.15 \\
\hline & NDUFB1 & 206790_s_at & OS & 836 & 281 & 0.85 & $0.64-1.12$ & 0.25 \\
\hline & NDUFS4 & 209303_at & OS & 333 & 784 & 0.82 & $0.64-1.05$ & 0.12 \\
\hline Ovarian & NDUFA1 & 202298_at & OS & 837 & 734 & 1.59 & $1.3-1.94$ & $6.4 \mathrm{E}-06$ \\
\hline \multirow[t]{3}{*}{ cancer } & NDUFA3 & 218563_at & OS & 1147 & 435 & 1.25 & $1.08-1.45$ & 0.0028 \\
\hline & NDUFA4 & 217773_s_at & OS & 1067 & 515 & 1.25 & $1.08-1.44$ & 0.002 \\
\hline & NDUFA6 & 202000_at & OS & 881 & 701 & 1.18 & $1.03-1.35$ & 0.016 \\
\hline
\end{tabular}




\begin{tabular}{|c|c|c|c|c|c|c|c|}
\hline NDUFA9 & 208969_at & OS & 748 & 834 & 1.23 & $1.08-1.41$ & 0.0022 \\
\hline NDUFA13 & 220864_s_at & OS & 949 & 633 & 1.17 & $1.03-1.34$ & 0.02 \\
\hline NDUFAF3 & 209177_at & OS & 1173 & 409 & 1.18 & $1.01-1.37$ & 0.034 \\
\hline NDUFB2 & 218201_at & OS & 500 & 1082 & 1.21 & $1.04-1.4$ & 0.011 \\
\hline NDUFB3 & 203371_s_at & OS & 1184 & 398 & 1.27 & $1.1-1.48$ & 0.0015 \\
\hline NDUFB4 & 218226_s_at & OS & 803 & 779 & 1.15 & $1-1.31$ & 0.044 \\
\hline NDUFB8 & 201227_s_at & OS & 780 & 802 & 1.23 & $1.07-1.4$ & 0.0027 \\
\hline NDUFS4 & 209303_at & OS & 1164 & 418 & 1.21 & $1.04-1.41$ & 0.016 \\
\hline NDUFS6 & 203606_at & OS & 462 & 1120 & 1.17 & $1-1.36$ & 0.045 \\
\hline NDUFA8 & 218160_at & OS & 401 & 1181 & 0.81 & $0.7-0.94$ & 0.0059 \\
\hline NDUFB6 & 203613_s_at & OS & 298 & 840 & 0.82 & $0.69-0.98$ & 0.028 \\
\hline NDUFA2 & 209224_s_at & OS & 1071 & 511 & 1.13 & $0.98-1.3$ & 0.084 \\
\hline NDUFAF4 & 219006_at & OS & 1163 & 419 & 1.14 & $0.98-1.33$ & 0.081 \\
\hline NDUFB5 & 203621_at & OS & 286 & 852 & 1.12 & $0.93-1.35$ & 0.22 \\
\hline NDUFB7 & 202839_s_at & OS & 704 & 878 & 1.11 & $0.97-1.27$ & 0.13 \\
\hline NDUFA5 & 201304_at & OS & 1175 & 407 & 1.12 & $0.96-1.3$ & 0.14 \\
\hline NDUFA7 & 202785_at & OS & 395 & 1187 & 0.9 & $0.77-1.04$ & 0.15 \\
\hline NDUFA10 & 217860_at & OS & 1135 & 447 & 0.9 & $0.77-1.05$ & 0.18 \\
\hline NDUFAF1 & 204125_at & OS & 437 & 1145 & 0.93 & $0.81-1.08$ & 0.36 \\
\hline NDUFB11 & 218320_s_at & OS & 1125 & 457 & 0.9 & $0.77-1.05$ & 0.17 \\
\hline NDUFC1 & 203478_at & OS & 587 & 995 & 0.91 & $0.8-1.04$ & 0.18 \\
\hline NDUFC2 & 218101_s_at & OS & 941 & 641 & 0.92 & $0.8-1.06$ & 0.23 \\
\hline
\end{tabular}




\begin{tabular}{|c|c|c|c|c|c|c|c|c|}
\hline & NDUFS5 & 201757_at & OS & 740 & 842 & 0.9 & $0.79-1.03$ & 0.12 \\
\hline & NDUFAB1 & 202077_at & OS & 592 & 990 & 1.06 & $0.92-1.21$ & 0.42 \\
\hline & NDUFB 1 & 206790_s_at & OS & 643 & 939 & 0.89 & $0.78-1.02$ & 0.081 \\
\hline & NDUFA11 & no available & OS & & & & & \\
\hline & NDUFA12 & & OS & & & & & \\
\hline & NDUFAF2 & & OS & & & & & \\
\hline & NDUFB9 & & OS & & & & & \\
\hline & NDUFB10 & & OS & & & & & \\
\hline & NDUFV3 & & OS & & & & & \\
\hline Lung & NDUFA1 & 202298_at & OS & 904 & 1022 & 1.24 & $1.09-1.41$ & 0.00093 \\
\hline \multirow[t]{12}{*}{ cancer } & NDUFA2 & 209223_at & OS & 671 & 1255 & 1.16 & $1.01-1.32$ & 0.032 \\
\hline & NDUFA3 & 218563_at & OS & 511 & 1415 & 1.57 & $1.34-1.84$ & $1.9 \mathrm{E}-08$ \\
\hline & NDUFA5 & 215850_s_at & OS & 1257 & 669 & 1.29 & $1.13-1.47$ & $1.4 \mathrm{E}-10$ \\
\hline & NDUFA7 & 202785_at & OS & 499 & 1427 & 1.38 & $1.18-1.62$ & $5.6 \mathrm{E}-05$ \\
\hline & NDUFA9 & 208969_at & OS & 1238 & 688 & 1.36 & $1.2-1.55$ & $2.3 \mathrm{E}-06$ \\
\hline & NDUFA10 & 217860_at & OS & 768 & 1158 & 1.55 & $1.35-1.78$ & $2.7 \mathrm{E}-10$ \\
\hline & NDUFA13 & 220864_s_at & OS & 500 & 1426 & 1.55 & $1.31-1.82$ & $1.8 E-07$ \\
\hline & NDUFAF3 & 209177_at & OS & 540 & 1386 & 1.51 & $1.29-1.76$ & $1.9 E-07$ \\
\hline & NDUFAF4 & 219006_at & OS & 1257 & 669 & 1.48 & $1.3-1.68$ & $1.4 \mathrm{E}-09$ \\
\hline & NDUFB1 & 206790_s_at & OS & 1369 & 557 & 1.17 & $1.02-1.34$ & 0.024 \\
\hline & NDUFB2 & 218201_at & OS & 645 & 1281 & 1.44 & $1.25-1.66$ & 5.30E-07 \\
\hline & NDUFB3 & 203371_s_at & OS & 485 & 1441 & 1.86 & $1.57-2.21$ & $7.1 \mathrm{E}-13$ \\
\hline
\end{tabular}




\begin{tabular}{|c|c|c|c|c|c|c|c|}
\hline NDUFB4 & 218226_s_at & OS & 484 & 1442 & 1.85 & $1.55-2.2$ & $2.9 \mathrm{E}-12$ \\
\hline NDUFB5 & 203621_at & OS & 1342 & 584 & 1.19 & $1.04-1.36$ & 0.0095 \\
\hline NDUFB6 & 203613_s_at & OS & 1144 & 782 & 1.31 & $1.15-1.49$ & $2.9 \mathrm{E}-05$ \\
\hline NDUFB7 & 202839_s_at & OS & 1007 & 919 & 1.31 & $1.15-1.48$ & $3.9 \mathrm{E}-05$ \\
\hline NDUFB8 & 214241_at & OS & 1163 & 763 & 1.23 & $1.08-1.4$ & 0.0016 \\
\hline NDUFB11 & 218320_s_at & OS & 939 & 987 & 1.24 & $1.09-1.41$ & 0.00089 \\
\hline NDUFC1 & 203478_at & OS & 771 & 1155 & 1.22 & $1.07-1.39$ & 0.0034 \\
\hline NDUFC2 & 218101_s_at & OS & 871 & 1055 & 1.43 & $1.25-1.63$ & 8.20E-08 \\
\hline NDUFS4 & 209303_at & OS & 544 & 1382 & 1.95 & $1.65-2.31$ & $2.6 \mathrm{E}-15$ \\
\hline NDUFS5 & 201757_at & OS & 1239 & 687 & 1.25 & $1.1-1.42$ & 0.00064 \\
\hline NDUFAF1 & 204125_at & OS & 1426 & 500 & 0.58 & $0.5-0.68$ & $9.1 \mathrm{E}-12$ \\
\hline NDUFS6 & 203606_at & OS & 1309 & 617 & 0.76 & $0.66-0.88$ & 0.00014 \\
\hline NDUFA4 & 217773_s_at & OS & 1442 & 484 & 1.09 & $0.95-1.26$ & 0.22 \\
\hline NDUFA6 & 202001_s_at & OS & 1328 & 598 & 1.08 & $0.95-1.24$ & 0.24 \\
\hline NDUFAB1 & 202077_at & OS & 592 & 990 & 1.06 & $0.92-1.21$ & 0.42 \\
\hline NDUFA8 & 218160_at & OS & 487 & 1439 & 0.92 & $0.79-1.06$ & 0.24 \\
\hline NDUFA11 & no available & OS & & & & & \\
\hline NDUFA12 & & OS & & & & & \\
\hline NDUFAF2 & & OS & & & & & \\
\hline NDUFB9 & & OS & & & & & \\
\hline NDUFB10 & & OS & & & & & \\
\hline NDUFV3 & & OS & & & & & \\
\hline
\end{tabular}




\begin{tabular}{|c|c|c|c|c|c|c|c|c|}
\hline Gastric & NDUFA1 & 202298_at & OS & 334 & 542 & 0.63 & $0.54-0.75$ & $1.5 E-07$ \\
\hline \multirow[t]{21}{*}{ cancer } & NDUFA2 & 209224_s_at & OS & 336 & 540 & 0.69 & $0.58-0.82$ & $2 E-05$ \\
\hline & NDUFA3 & 218563_at & OS & 437 & 439 & 0.8 & $0.68-0.95$ & 0.012 \\
\hline & NDUFA4 & 217773_s_at & OS & 237 & 639 & 0.76 & $0.63-0.92$ & 0.0039 \\
\hline & NDUFA5 & 201304_at & OS & 486 & 390 & 0.5 & $0.42-0.6$ & $1.6 \mathrm{E}-14$ \\
\hline & NDUFA6 & 202001_s_at & OS & 623 & 253 & 0.69 & $0.57-0.84$ & 0.00018 \\
\hline & NDUFA7 & 202785_at & OS & 587 & 289 & 0.5 & $0.41-0.61$ & $2.2 \mathrm{E}-12$ \\
\hline & NDUFA8 & 218160_at & OS & 352 & 524 & 0.6 & $0.51-0.71$ & $3.8 \mathrm{E}-09$ \\
\hline & NDUFA13 & 220864_s_at & OS & 454 & 422 & 0.64 & $0.54-0.76$ & $3.3 E-07$ \\
\hline & NDUFAF1 & 204125_at & OS & 651 & 225 & 0.5 & $0.41-0.63$ & $2.1 \mathrm{E}-10$ \\
\hline & NDUFAF3 & 209177_at & OS & 242 & 634 & 0.75 & $0.62-0.9$ & 0.0022 \\
\hline & NDUFAF4 & 219006_at & OS & 600 & 276 & 0.6 & $0.49-0.73$ & 2.20E-07 \\
\hline & NDUFAB1 & 202077_at & OS & 564 & 312 & 0.67 & $0.56-0.81$ & $2.2 \mathrm{E}-05$ \\
\hline & NDUFB1 & 206790_s_at & OS & 417 & 459 & 0.73 & $0.62-0.87$ & 0.00029 \\
\hline & NDUFB2 & 218200_s_at & OS & 346 & 530 & 0.66 & $0.56-0.78$ & $1.7 E-06$ \\
\hline & NDUFB3 & 203371_s_at & OS & 656 & 220 & 0.65 & $0.53-0.81$ & 0.0001 \\
\hline & NDUFB4 & 218226_s_at & OS & 496 & 380 & 0.75 & $0.63-0.89$ & 0.0011 \\
\hline & NDUFB5 & 203621_at & OS & 515 & 361 & 0.61 & $0.51-0.73$ & $6.4 E-08$ \\
\hline & NDUFB6 & 203613_s_at & OS & 551 & 325 & 0.49 & $0.41-0.6$ & $8.5 E-14$ \\
\hline & NDUFB7 & 202839_s_at & OS & 473 & 403 & 0.56 & $0.47-0.66$ & $3 E-11$ \\
\hline & NDUFB8 & 201226_at & OS & 250 & 626 & 0.71 & $0.59-0.85$ & 0.00022 \\
\hline & NDUFB11 & 218320_s_at & OS & 428 & 448 & 0.73 & $0.62-0.86$ & 0.00026 \\
\hline
\end{tabular}




\begin{tabular}{|c|c|c|c|c|c|c|c|}
\hline NDUFC1 & 203478_at & OS & 472 & 472 & 0.55 & $0.46-0.66$ & $1.1 \mathrm{E}-11$ \\
\hline NDUFC2 & 218101_s_at & OS & 652 & 224 & 0.79 & $0.64-0.97$ & 0.021 \\
\hline NDUFS4 & 209303_at & OS & 457 & 419 & 0.65 & $0.55-0.77$ & $5.2 E-07$ \\
\hline NDUFS6 & 203606_at & OS & 429 & 447 & 0.78 & $0.66-0.92$ & 0.0037 \\
\hline NDUFA10 & 217860_at & OS & 305 & 571 & 0.93 & $0.78-1.11$ & 0.4 \\
\hline NDUFS5 & 201757_at & OS & 628 & 248 & 1.26 & $1.05-1.51$ & 0.014 \\
\hline NDUFA9 & 208969_at & OS & 367 & 509 & 1.12 & $0.94-1.33$ & 0.19 \\
\hline NDUFA11 & no available & OS & & & & & \\
\hline NDUFA12 & & OS & & & & & \\
\hline NDUFAF2 & & OS & & & & & \\
\hline NDUFB9 & & OS & & & & & \\
\hline NDUFB10 & & OS & & & & & \\
\hline NDUFV3 & & OS & & & & & \\
\hline
\end{tabular}


S-Table 5. The summary of desired Affymetrix ID, Cases Number, Hazard Ratio (HR; and $95 \%$ confidence intervals) and Log Rank P of the individual core gene's other survival curves for the four different tumors.

\section{Cases-1 Cases}

Subunits Affymetrix ID Survival ow -high HR 95\% CI P-value

\begin{tabular}{|c|c|c|c|c|c|c|c|c|}
\hline Breast & NDUFS1 & 203039_s_at & RFS & 1809 & 1745 & 1.63 & $1.45-1.83$ & 0 \\
\hline \multirow[t]{11}{*}{ cancer } & NDUFS2 & 208969_at & RFS & 2494 & 1060 & 1.29 & $1.14-1.46$ & $3.20 \mathrm{E}-05$ \\
\hline & NDUFS3 & 201740_at & RFS & 1753 & 1801 & 1.26 & $1.12-1.41$ & $7.50 \mathrm{E}-05$ \\
\hline & NDUFS7 & 211752_s_at & RFS & 2059 & 1495 & 1.22 & $1.09-1.37$ & 0.00058 \\
\hline & NDUFS8 & 203189_s_at & RFS & 1771 & 1783 & 1.35 & $1.2-1.52$ & $2.40 \mathrm{E}-07$ \\
\hline & NDUFS8 & 203190_at & RFS & 1809 & 1745 & 1.43 & $1.28-1.6$ & $6.40 \mathrm{E}-10$ \\
\hline & NDUFV1 & 208714_at & RFS & 2376 & 1178 & 1.29 & $1.15-1.45$ & $1.80 \mathrm{E}-05$ \\
\hline & NDUFS2 & 208969_at & PPS & 252 & 99 & 1.44 & $1.09-1.89$ & $1.00 \mathrm{E}-02$ \\
\hline & NDUFS3 & 201740_at & PPS & 153 & 198 & 0.64 & $0.5-0.83$ & 0.00074 \\
\hline & NDUFV2 & 202941_at & PPS & 91 & 260 & 0.61 & $0.46-0.8$ & 0.00042 \\
\hline & NDUFS1 & 203039_s_at & DMFS & 1160 & 449 & 1.46 & $1.18-1.81$ & $5.00 \mathrm{E}-04$ \\
\hline & NDUFS2 & 208969_at & DMFS & 1134 & 475 & 1.28 & $1.04-1.59$ & 0.021 \\
\hline Ovarian & NDUFS1 & 203039_s_at & PFS & 373 & 933 & 1.23 & $1.07-1.42$ & 0.0039 \\
\hline \multirow[t]{4}{*}{ cancer } & NDUFS3 & 201740_at & PFS & 775 & 531 & 0.84 & $0.73-0.96$ & 0.011 \\
\hline & NDUFS7 & 211752_s_at & PFS & 359 & 947 & 0.81 & $0.69-0.93$ & 0.004 \\
\hline & NDUFV2 & 202941_at & PFS & 344 & 962 & 0.77 & $0.67-0.89$ & 0.00035 \\
\hline & NDUFS8 & 203189_s_at & PPS & 325 & 383 & 0.76 & $0.63-0.9$ & 0.76 \\
\hline
\end{tabular}




\begin{tabular}{|c|c|c|c|c|c|c|c|c|}
\hline & NDUFV2 & 202941_at & PPS & 269 & 439 & 0.82 & $0.68-0.98$ & 0.029 \\
\hline Lung & NDUFS1 & 203039_s_at & FP & 668 & 314 & 0.7 & $0.57-0.87$ & 0.0013 \\
\hline \multirow[t]{8}{*}{ cancer } & NDUFS2 & 201966_at & $\mathrm{FP}$ & 310 & 672 & 1.24 & $1-1.53$ & 0.051 \\
\hline & NDUFS3 & 201740_at & $\mathrm{FP}$ & 261 & 721 & 1.74 & $1.36-2.21$ & $5.80 \mathrm{E}-06$ \\
\hline & NDUFS7 & 211752_s_at & $\mathrm{FP}$ & 344 & 638 & 1.31 & $1.06-1.61$ & 0.011 \\
\hline & NDUFS8 & 203189_s_at & FP & 281 & 701 & 1.8 & $1.42-2.27$ & $6.00 \mathrm{E}-07$ \\
\hline & NDUFV1 & 208714_at & FP & 715 & 267 & 1.24 & $1.01-1.53$ & 0.039 \\
\hline & NDUFV2 & 202941_at & FP & 690 & 292 & 1.72 & $1.41-2.1$ & $5.00 \mathrm{E}-08$ \\
\hline & NDUFS8 & 203189_s_at & PPS & 257 & 87 & 1.6 & $1.21-2.11$ & 0.00079 \\
\hline & NDUFV1 & 208714_at & PPS & 226 & 118 & 1.5 & $1.16-1.95$ & 0.0022 \\
\hline Gastric & NDUFS1 & 203039_s_at & $\mathrm{FP}$ & 427 & 214 & 0.52 & $0.41-0.66$ & $5.60 \mathrm{E}-08$ \\
\hline \multirow[t]{5}{*}{ cancer } & NDUFS2 & 201966_at & FP & 293 & 348 & 0.69 & $0.56-0.84$ & 0.00023 \\
\hline & NDUFS3 & 201740_at & FP & 376 & 265 & 0.58 & $0.47-0.72$ & $3.80 \mathrm{E}-07$ \\
\hline & NDUFS7 & 211752_s_at & FP & 403 & 238 & 0.59 & $0.47-0.73$ & $1.50 \mathrm{E}-06$ \\
\hline & NDUFS8 & 203189_s_at & FP & 164 & 477 & 0.76 & $0.61-0.94$ & 0.013 \\
\hline & NDUFV2 & 202941_at & FP & 261 & 380 & 0.6 & $0.49-0.73$ & $4.90 \mathrm{E}-07$ \\
\hline
\end{tabular}


S-Table 6. The "all genes" mRNA expression in different tumor types from Oncomine.

Screening conditions

\begin{tabular}{|c|c|c|c|c|c|c|c|c|}
\hline Subunits & Analysis Type & type & hold & ) & old & -ets & datasets & datasets \\
\hline NDUFA1 & cancer VS normal & mRNA & 0.01 & $10 \%$ & 2 & 327 & 10 & 6 \\
\hline NDUFA2 & cancer VS normal & mRNA & 0.01 & $10 \%$ & 2 & 292 & 20 & 2 \\
\hline NDUFA3 & cancer VS normal & mRNA & 0.01 & $10 \%$ & 2 & 301 & 17 & 5 \\
\hline NDUFA4 & cancer VS normal & mRNA & 0.01 & $10 \%$ & 2 & 316 & 11 & 13 \\
\hline NDUFA5 & cancer VS normal & mRNA & 0.01 & $10 \%$ & 2 & 351 & 10 & 9 \\
\hline NDUFA6 & cancer VS normal & mRNA & 0.01 & $10 \%$ & 2 & 280 & 17 & 6 \\
\hline NDUFA7 & cancer VS normal & mRNA & 0.01 & $10 \%$ & 2 & 336 & 14 & 5 \\
\hline NDUFA8 & cancer VS normal & mRNA & 0.01 & $10 \%$ & 2 & 250 & 14 & 5 \\
\hline NDUFA9 & cancer VS normal & mRNA & 0.01 & $10 \%$ & 2 & 351 & 18 & 1 \\
\hline NDUFA10 & cancer VS normal & mRNA & 0.01 & $10 \%$ & 2 & 293 & 8 & 5 \\
\hline NDUFA11 & cancer VS normal & mRNA & 0.01 & $10 \%$ & 2 & 194 & 11 & 2 \\
\hline NDUFA12 & cancer VS normal & mRNA & 0.01 & $10 \%$ & 2 & 199 & 10 & 0 \\
\hline NDUFA13 & cancer VS normal & mRNA & 0.01 & $10 \%$ & 2 & 295 & 16 & 4 \\
\hline NDUFAF1 & cancer VS normal & mRNA & 0.01 & $10 \%$ & 2 & 325 & 6 & 11 \\
\hline
\end{tabular}




\begin{tabular}{|c|c|c|c|c|c|c|c|c|}
\hline NDUFAF2 & cancer VS normal & mRNA & 0.01 & $10 \%$ & 2 & 181 & 12 & 2 \\
\hline NDUFAF3 & cancer VS normal & mRNA & 0.01 & $10 \%$ & 2 & 295 & 9 & 10 \\
\hline NDUFAF4 & cancer VS normal & mRNA & 0.01 & $10 \%$ & 2 & 283 & 8 & 7 \\
\hline NDUFAB1 & cancer VS normal & mRNA & 0.01 & $10 \%$ & 2 & 296 & 8 & 0 \\
\hline NDUFB1 & cancer VS normal & mRNA & 0.01 & $10 \%$ & 2 & 294 & 13 & 3 \\
\hline NDUFB2 & cancer VS normal & mRNA & 0.01 & $10 \%$ & 2 & 295 & 14 & 3 \\
\hline NDUFB3 & cancer VS normal & mRNA & 0.01 & $10 \%$ & 2 & 304 & 7 & 1 \\
\hline NDUFB4 & cancer VS normal & mRNA & 0.01 & $10 \%$ & 2 & 267 & 7 & 4 \\
\hline NDUFB5 & cancer VS normal & mRNA & 0.01 & $10 \%$ & 2 & 331 & 13 & 0 \\
\hline NDUFB6 & cancer VS normal & mRNA & 0.01 & $10 \%$ & 2 & 333 & 6 & 2 \\
\hline NDUFB7 & cancer VS normal & mRNA & 0.01 & $10 \%$ & 2 & 363 & 19 & 4 \\
\hline NDUFB8 & cancer VS normal & mRNA & 0.01 & $10 \%$ & 2 & 305 & 11 & 10 \\
\hline NDUFB9 & cancer VS normal & mRNA & 0.01 & $10 \%$ & 2 & 162 & 18 & 2 \\
\hline NDUFB10 & cancer VS normal & mRNA & 0.01 & $10 \%$ & 2 & 220 & 6 & 4 \\
\hline NDUFB11 & cancer VS normal & mRNA & 0.01 & $10 \%$ & 2 & 268 & 20 & 2 \\
\hline NDUFC1 & cancer VS normal & mRNA & 0.01 & $10 \%$ & 2 & 322 & 12 & 7 \\
\hline NDUFC2 & cancer VS normal & mRNA & 0.01 & $10 \%$ & 2 & 270 & 11 & 1 \\
\hline NDUFS4 & cancer VS normal & mRNA & 0.01 & $10 \%$ & 2 & 338 & 5 & 5 \\
\hline NDUFS5 & cancer VS normal & mRNA & 0.01 & $10 \%$ & 2 & 333 & 17 & 2 \\
\hline NDUFS6 & cancer VS normal & mRNA & 0.01 & $10 \%$ & 2 & 315 & 18 & 3 \\
\hline NDUFV3 & cancer VS normal & mRNA & 0.01 & $10 \%$ & 2 & 237 & 6 & 6 \\
\hline
\end{tabular}


S-Table 7. Order of the studies in Fig4A and Fig4C (from left to right)

\begin{tabular}{|c|c|c|}
\hline Order & ALL genes & Core genes \\
\hline 1 & Breast(BCCRC Xenograft) & Breast(BCCRC Xenograft) \\
\hline 2 & Ovarian(TCGA) & Pancreas (UTSW) \\
\hline 3 & Lung squ(TCGA) & Bladder(TCGA pub) \\
\hline 4 & Pancreas (UTSW) & Bladder(TCGA) \\
\hline 5 & Ovarian(TCGA pub) & Breast(TCGA) \\
\hline 6 & Esophagus(TCGA) & Esophagus(TCGA) \\
\hline 7 & NCI-60 & Liver(TCGA) \\
\hline 8 & Lung squ(TCGA pub) & Ovarian(TCGA) \\
\hline 9 & CCLE(Novartis/Broad 2012) & NCI-60 \\
\hline 10 & Bladder(TCGA) & Uterine(TCGA) \\
\hline 11 & Bladder(TCGA pub) & Gastric(TCGA) \\
\hline 12 & Prostate(MICH) & CCLE(Novartis/Broad 2012) \\
\hline 13 & Head \& neck(TCGA pub) & Lung adeno(TCGA) \\
\hline 14 & Head \& neck(TCGA) & MPNST(MSKCC) \\
\hline 15 & Lung adeno(TCGA pub) & Uterine(TCGA) \\
\hline 16 & Uterine CS(TCGA) & Gastric(TCGA pub) \\
\hline 17 & Lung adeno(TCGA) & Bladder(MSKCC 2012) \\
\hline 18 & Breast（TCGA pub2015） & Sarcoma(TCGA) \\
\hline 19 & Sarcoma(TCGA) & Lung adeno(TCGA pub) \\
\hline 20 & Breast(TCGA) & Head \& neck(TCGA pub) \\
\hline
\end{tabular}


21 Gastric(TCGA pub)

22 Gastric(TCGA)

23 Cervical(TCGA)

24 Prostate(SU2C)

25 Melanoma(TCGA)

26 MPNST(MSKCC)

27 Liver(TCGA)

28 Breast(TCGA pub)

29 DLBC(TCGA)

30 Uterine(TCGA)

31 ACyCle(MSKCC)

32 Uterine(TCGA pub)

33 Prostate(TCGA)

34 ACC(TCGA)

35 Prostate(TCGA 2015)

$36 \operatorname{ccRCC}(\mathrm{TCGA})$

37 Pancrease(TCGA)

38 Lung adeno(Broad)

39 Glioma(TCGA)

40 Uveal Melanoma（TCGA）

41 Bladder(MSKCC 2012)

42 Sarcoma(MSKCC)
ACyCle(MSKCC)

Lung squ(TCGA)

Breast(TCGA pub)

Head \& neck(TCGA)

Melanoma(TCGA)

Prostate(SU2C)

ucs（Johns Hopkins 2014）

Cervical(TCGA)

Uterine CS(TCGA)

Ovarian(TCGA pub)

Sarcoma(MSKCC)

Lung squ(TCGA pub)

Lung adeno(Broad)

Prostate(MICH)

Pancrease(TCGA)

DLBC(TCGA)

Colorectal(TCGA pub)

Colorectal(TCGA)

PCPG(TCGA)

Prostate(TCGA)

Melanoma(Yale)

Prostate(TCGA 2015) 


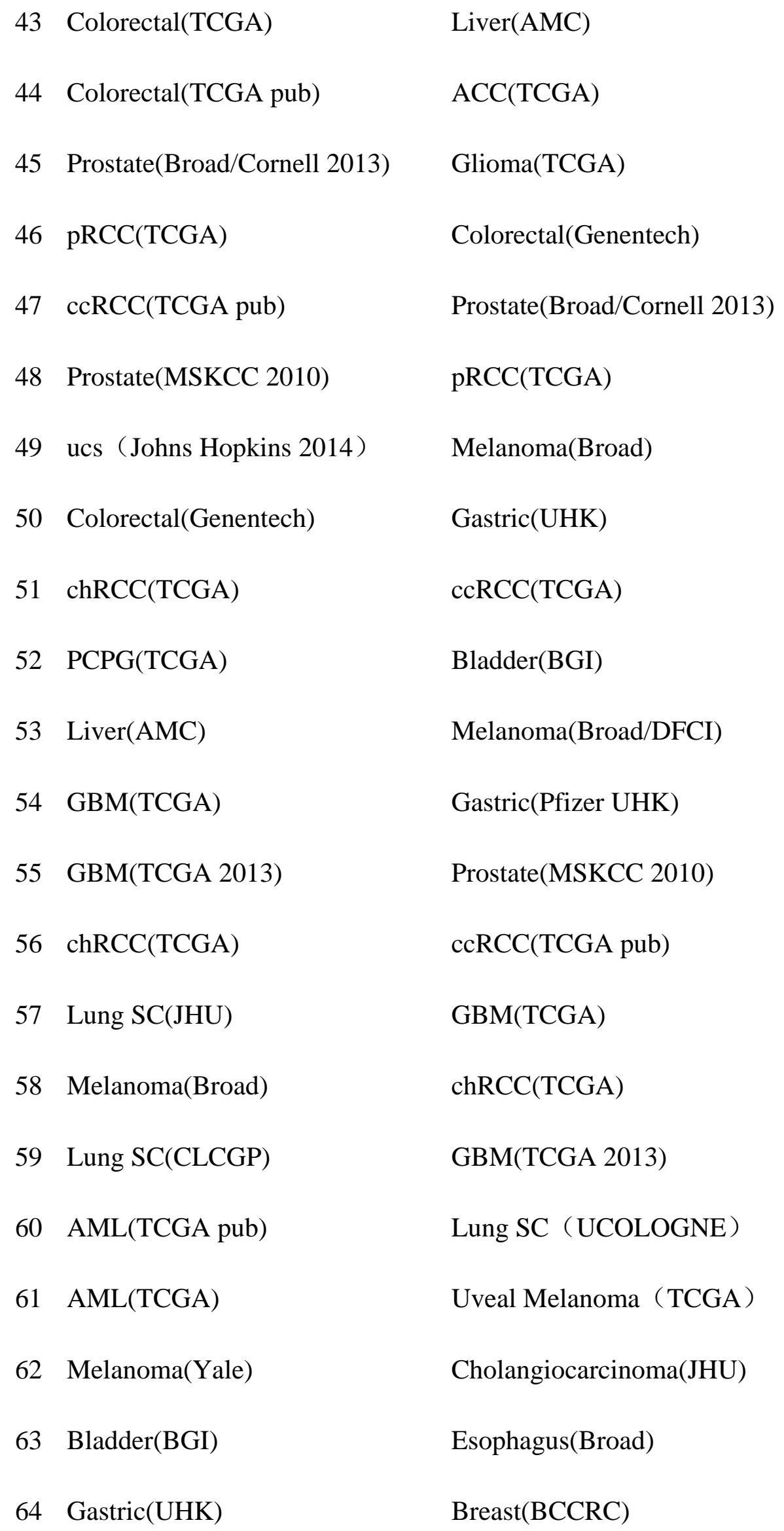


65 Head \& neck(Broad)

66 Melanoma(Broad/DFCI)

67 Gastric(Pfizer UHK)

68 Esophagus(ICGC)

69 Esophagus(Broad)

70 Lung SC (UCOLOGNE)

71 Ewing sarcoma (DFCI)

72 Breast(Sanger)

73 Thyriod(TCGA pub)

74 Thyriod(TCGA)

75 Breast(Broad)

76 GBM(TCGA 2008)

77 Prostate(Broad/Cornell 2012)

78 Cholangiocarcinoma(JHU)

79 Prostate(MSKCC 2014)

80 NPC(Singapore)

81 Breast(BCCRC)

$82 \mathrm{MM}($ Broad $)$

83 nccRCC (genentech 2014)

$84 \operatorname{ccRCC}(\mathrm{BGI})$

85 Pancreas(ICGC)

86 MBL(ICGC)
chRCC(TCGA)

Head \& neck(Broad)

Esophagus(ICGC)

AML(TCGA)

AML(TCGA pub)

$\operatorname{ccRCC}(\mathrm{BGI})$

Thyriod(TCGA pub)

Thyriod(TCGA)

Breast(Broad)

Prostate(MSKCC 2014)

Ewing sarcoma（DFCI）

Prostate(Broad/Cornell 2012)

MBL(ICGC)

nccRCC（genentech 2014）

Breast(sanger) 